\title{
Probing turbulence with infrared observations in OMC1 ${ }^{\star}$
}

\author{
M. Gustafsson ${ }^{1}$, D. Field ${ }^{1}$, J. L. Lemaire ${ }^{2}$, and F. P. Pijpers ${ }^{3}$ \\ 1 Department of Physics and Astronomy, University of Aarhus, 8000 Aarhus C, Denmark \\ e-mail: maikeng@phys.au.dk \\ 2 Observatoire de Paris \& Université de Cergy-Pontoise, LERMA \& UMR 8112 du CNRS, 92195 Meudon, France \\ 3 Space and Atmospheric Physics, Dept. Physics, Imperial College London, England
}

Received 16 May 2005 / Accepted 8 September 2005

ABSTRACT

A statistical analysis is presented of the turbulent velocity structure in the Orion Molecular Cloud at scales ranging from $70 \mathrm{AU}$ to $3 \times 10^{4} \mathrm{AU}$. Results are based on IR Fabry-Perot interferometric observations of shock and photon-excited $\mathrm{H}_{2}$ in the $K$-band $\mathrm{S}(1) v=1-0$ line at $2.121 \mu \mathrm{m}$ and refer to the dynamical characteristics of warm perturbed gas. Data consist of a spatially resolved image with a measured velocity for each resolution limited region $(70 \mathrm{AU} \times 70 \mathrm{AU})$ in the image. The effect of removal of apparent large scale velocity gradients is discussed and the conclusion drawn that these apparent gradients represent part of the turbulent cascade and should remain within the data. Using our full data set, observations establish that the Larson size-linewidth relation is obeyed to the smallest scales studied here extending the range of validity of this relationship by nearly 2 orders of magnitude. The velocity probability distribution function (PDF) is constructed showing extended exponential wings, providing evidence of intermittency, further supported by the skewness (third moment) and kurtosis (fourth moment) of the velocity distribution. Variance and kurtosis of the PDF of velocity differences are constructed as a function of lag. The variance shows an approximate power law dependence on lag, with exponent significantly lower than the Kolmogorov value, and with deviations below $2000 \mathrm{AU}$ which are attributed to outflows and possibly disk structures associated with low mass star formation within OMC1. The kurtosis shows strong deviation from a Gaussian velocity field, providing evidence of velocity correlations at small lags. Results agree accurately with semi-empirical simulations in Eggers \& Wang (1998).

In addition, 170 individual $\mathrm{H}_{2}$ emitting clumps have been analysed with sizes between 500 and 2200 AU. These show considerable diversity with regard to PDFs and variance functions (related to second order structure functions) displaying a variety of shapes of the PDF and different values of the scaling exponent within a restricted spatial region. However, a region associated with an outflow from a deeply embedded O-star shows high values of the scaling exponent of the variance function, representing a strong segregation of high and low exponent clumps. Our analysis constitutes the first characterization of the turbulent velocity field at the scale of star formation and provide a dataset which models of star-forming regions should aim to reproduce.

Key words. ISM: individual objects: OMC1 - ISM: kinematics and dynamics - ISM: molecules - shock waves - turbulence - infrared: ISM

\section{Introduction}

A key to understanding the mechanism of star formation is to characterise in detail the nature of the turbulent, weakly ionized and magnetised plasma in which stars form. Recently Gustafsson et al. 2003 (Paper I) published observational results for the vibrational emission of $\mathrm{H}_{2}$ in the archetypal star-forming region in the Orion Molecular Cloud, OMC1. Using a combination of Fabry-Perot interferometry and the PUEO adaptive optics system on the Canada-France-Hawaii Telescope (CFHT), data achieved a spatial resolution of 0.15 (70 AU at the distance of OMC1, $D=460 \mathrm{pc}$ (Bally et al. 2000)), with a velocity discrimination of $1 \mathrm{~km} \mathrm{~s}^{-1}$ in regions

* Based on observations obtained at the Canada-France-Hawaii Telescope (CFHT) which is operated by the National Research Council of Canada, the Institut National des Science de l'Univers of the Centre National de la Recherche Scientifique of France, and the University of Hawaii. of high brightness. These data, limited as they are to highly excited regions in OMC1, provide the first opportunity to study the physical properties of a star-forming region both in terms of its morphology and bulk gas motion at the scale of star formation. The aim of the present paper is to characterise the nature of the turbulent velocity field in OMC1 for the subset of regions represented through vibrationally excited $\mathrm{H}_{2}$. Our results should help to provide a benchmark for comparison with MHD models of star-forming regions.

The importance of turbulent gas motion in regulating star formation may be illustrated as follows. Relative bulk motions obtained from observations reported here range up to $40 \mathrm{~km} \mathrm{~s}^{-1}$ in dense gas. Since these motions are supersonic, it is evident that bulk motion must contribute more energy per unit volume, that is, pressure, than thermal energy. Equally if a simple scaling law is used between magnetic induction, $B$, and particle density, $n$, whereby $B=b n^{1 / 2} \mu \mathrm{G}$, with $n$ in $\mathrm{cm}^{-3}$ 
(Troland \& Heiles 1986), then the energy contained in bulk mass motion will exceed the energy in the magnetic field, $B^{2} / 2 \mu$ (in SI units), for bulk velocities greater than $2.2 \mathrm{~b} \mathrm{~km} \mathrm{~s}^{-1}$. The constant $\mathrm{b}$ lies typically between 1 and 2 (Kristensen et al. 2005). Thus in many regions the turbulent pressure may exceed the magnetic pressure, given that bulk motion proceeds at tens of $\mathrm{km} \mathrm{s}^{-1}$ as mentioned above. The magnetic pressure in turn typically exceeds the thermal pressure, the ratio of thermal to magnetic pressure being $\sim 5 \times 10^{-3} T / b^{2}$, where $T$ is the gas temperature. In the above simple prescription, any anisotropy of the magnetic field has been ignored. At all events, on purely energetic grounds, in earlier stages of star formation, turbulence is the controlling support mechanism against gravitational collapse, if bulk mass motions exceed a few $\mathrm{km} \mathrm{s}^{-1}$. This statement remains true even in the warm regions involved in the present study.

Observational and computational evidence for the simple picture presented above has grown considerably in the last decade as described in recent reviews by Larson (2003) and Mac Low \& Klessen (2004). Moreover the role of turbulence goes further than simply slowing the process of gravitational collapse. Simulations show that turbulence may determine the initial mass function (IMF) for star formation through turbulent fragmentation (e.g. Nordlund \& Padoan 2003). It is evident that MHD models, which make such far-reaching predictions, need to be constrained by observations. The meeting of theory and observation may be achieved by recording the statistical properties of velocity fields and this standard approach is adopted here. In the present work we make only very limited comparison with published models. Rather, we present modellers with characteristic behaviour which it is their challenge to reproduce.

Several techniques have previously been used to characterize the structure of brightness and velocity in molecular clouds based largely upon CO observations, reviewed by Goodman et al. (1998) and Miesch et al. (1999). These earlier observations, tracing relatively cool and low density gas, are limited in spatial resolution and can only be used to address the physics at scales larger than roughly $0.03 \mathrm{pc}$ (6000 AU). When dealing with turbulence it is assumed that most features are scalefree between the driving and dissipation scale and that the behaviour for example of the structure functions (see Sect. 4.4) can be extrapolated to the very much smaller scales of individual star formation. This is however far from certain. The high spatial resolution infrared data reported in Paper I give the opportunity to discover how structure functions develop at much smaller scales than have hitherto been probed.

In this paper we use three standard statistical measures to provide observational constraints on theories and simulations concerning gravitational collapse and star formation in a turbulent ISM. First, we test whether the observed scaling behaviour of the velocity structure, encapsulated in the sizelinewidth relation or "Larson law" (Larson 1981), holds for the smaller scales involved here (Sect. 4.2). Second, we use probability distribution functions (PDFs) of velocities (Sect. 4.3) as a probe of intermittency (Falgarone \& Phillips 1990, 1991; Falgarone et al. 1994; Miesch \& Scalo 1995; Miesch et al. 1999; Ossenkopf \& Mac Low 2002). Third, we calculate the low order moments of the velocity difference PDF (Miesch \& Scalo 1995; Lis et al. 1998; Miesch et al. 1999; Ossenkopf \& Mac Low 2002) as a function of lag (Sect. 4.4), that is, the structure functions or functions directly related to structure functions. In addition, the high spatial resolution of the data allows us to go further than determining these quantities for the observed field as a whole. In Sect. 5 we identify 170 individual clumps in OMC1 with a mean size of 1300 AU. We calculate the individual PDFs of peak velocities and the variance functions for these clumps.

\section{Observations and data reduction}

Near infrared $K$-band observations of the strongest $\mathrm{H}_{2}$ emission line in Orion, $v=1-0 \mathrm{~S}(1)$ at $2.121 \mu \mathrm{m}$, are used as a tracer of radial gas velocity to provide the data for the statistical analysis performed here. The observational data are the same as those described in Paper I and a detailed description of the data acquisition and reduction may be found there. In brief, OMC1 was observed using the CFHT with GriF on the night of December 5th 2000. The GriF instrument (Clénet et al. 2002), is a combination of the PUEO adaptive optics (AO) system on the CFHT with interferometric spectral scanning. The interferometer, a Queensgate ET50WF Fabry-Perot (FP), affords a measured spectral resolution $\lambda / \Delta \lambda \sim 2030$, that is, $150 \mathrm{~km} \mathrm{~s}^{-1}$. The detector has a field of view of $36^{\prime \prime} \times 36^{\prime \prime}$ with a pixel scale of $0{ }^{\prime} 035$. The region of OMC1 observed, shown in Fig. 1, consists of four overlapping fields and the entire region is centred $15^{\prime \prime} \mathrm{N}$ and $15^{\prime \prime} \mathrm{W}$ of TCC0016 $\left(05^{\mathrm{h}} 35^{\mathrm{m}} 14^{\mathrm{s}} .91,-05^{\circ} 22^{\prime} 39^{\prime} \cdot 31\right.$, J2000).

Each field has been scanned through the $\mathrm{H}_{2}$ line from a wavelength in the far blue wing to a wavelength in the far red wing, using step sizes between $4.4 \times 10^{-4} \mu \mathrm{m}$ and $4.6 \times 10^{-4} \mu \mathrm{m}$ $\left(\sim 65 \mathrm{~km} \mathrm{~s}^{-1}\right)$, allowing adequate sampling of the instrumental profile. To prevent superposition of different FP orders during scanning, a $\mathrm{H}_{2} v=1-0 \mathrm{~S}(1)$ interference filter with a $2.122 \mu \mathrm{m}$ central wavelength and a bandwidth of $0.02 \mu \mathrm{m}$, was inserted between the FP and the detector. For each region and each wavelength, a single exposure of $400 \mathrm{~s}$ was performed. Data reduction includes dark and bias subtraction, flat-fielding, bad pixel rejection, image recentering, 2D wavelength correction and subtraction of sky background obtained from an image in the far wing of the $\mathrm{H}_{2}$ profile. Various reference stars for the AO were used: TCC0016 $\left(m_{V} \sim 14\right)$ in the SE and SW field, Parenago $1838\left(m_{V} \sim 8\right)$ in NE and Parenago $1819\left(m_{V} \sim 12\right)$ in NW. The FWHM of the point-spread-function of stars in the region has been examined yielding a spatial resolution of 0.15 . This near diffraction limited spatial resolution was maintained throughout all observations. In view of the spatial resolution of 0 .' 15 , all images have been smoothed by a moving average of 3 by 3 pixels to improve $\mathrm{S} / \mathrm{N}$ without significantly degrading the spatial resolution.

Radial velocities were found by choosing a specific position on the sky and taking a cut through the cube made up of the channel maps. This yielded a set of count rates as a function of wavelength constituting a line profile and a Lorentzian is fitted to the profile (Clénet et al. 2002). The position of the peak in emission gives the radial velocity. The Lorentzian form 
N

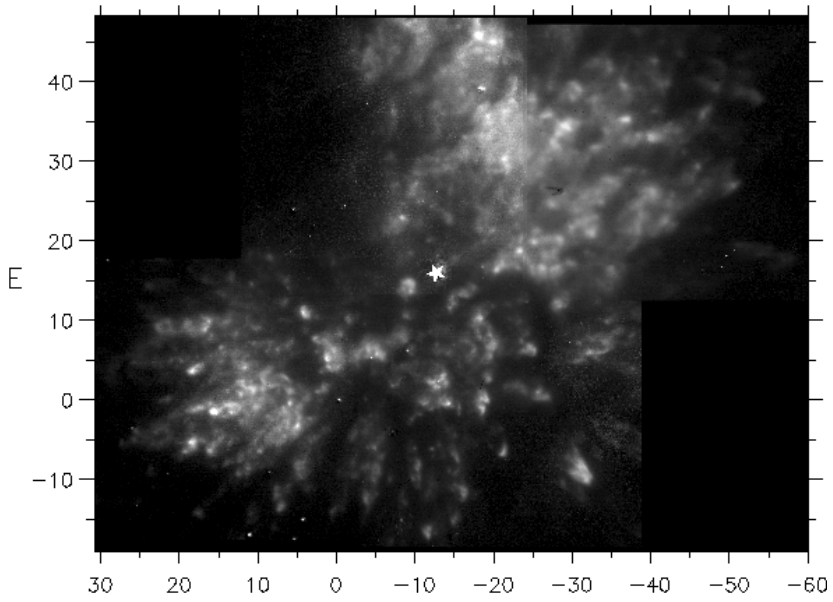

Fig. 1. Velocity integrated emission in the $\mathrm{S}(1) v=1-0 \mathrm{H}_{2}$ emission line covering the full observed field in OMC1. $(0,0)$ marks the position of TCC0016 $05^{\mathrm{h}} 35^{\mathrm{m}} 14.91,-05^{\circ} 22^{\prime} 399^{\prime} 31$ (J2000). The star marks the position of BN. Units are arcseconds, where $1 \operatorname{arcsec}=460 \mathrm{AU}$.

was chosen because it represents the instrumental profile of the Fabry-Perot. We note that the instrument profile is highly symmetric (Clénet et al. 2002) and thus a choice for example of a centroid rather than the peak of a Lorentzian is immaterial to an estimation of the velocity. We also note that less than $2 \%$ of profiles are double peaked: the velocity of the brighter of the two peaks is chosen when these cases are encountered (Paper I).

Performing these operations pixel by pixel for the full field of view results in very accurate fits for the brighter regions. Including statistical error in the count rates, relative peak wavelengths may be found in bright local zones to 2 to 3 parts per $10^{6}(3 \sigma)$, that is, to better than $\pm 1 \mathrm{~km} \mathrm{~s}^{-1}$. The quality of the fit decreases with decreasing brightness. Since an assessment of errors is important in discussing the statistical properties of the velocity field, the accuracy of determination of the central wavelength has been estimated by performing a large number of fits for a range of pixel brightness. An empirical correspondence between brightness and error in velocity has thereby been determined. This may be expressed as follows:

$$
\begin{aligned}
\sigma= & 0.15+18.1 \exp \left(-I / 4.8 \times 10^{-2}\right) \\
& +2.15 \exp (-I / 1.2))
\end{aligned}
$$

where $\sigma$ is the standard deviation of the radial velocity in $\mathrm{km} \mathrm{s}^{-1}$ and $I$ is the brightness in counts pixel ${ }^{-1} \mathrm{~s}^{-1}$ for the peak emission associated with the Lorentzian fit. All pixels with brightness below 0.05 counts pixel $^{-1} \mathrm{~s}^{-1}$, that is $2 \%$ of the maximum brightness, have been excluded. Thus the largest uncertainty, $\sigma$, in the radial velocity that may be encountered in our data is $8.6 \mathrm{~km} \mathrm{~s}^{-1}$.

In addition to random errors in the velocity, systematic errors may also occur in establishing velocity differences between regions which are physically remote. This may take place due to distortions in the $2 \mathrm{D}$ wavelength correction arising from possible mechanical instabilities or drift. In this connection, observations of a restricted part of the full field comprising two $36^{\prime \prime} \times 36^{\prime \prime}$ fields to the SE and NW were performed in January 2003. Comparisons have been made of the velocity fields in the 2000 data, used here, and in the 2003 data. Average velocities have been estimated within regions of about $50 \times 50$ pixels $\left(1^{\prime \prime} .75 \times 11^{\prime \prime} .75\right)$ separated by up to $60^{\prime \prime}$, the full size of the image. We find that velocity differences in the 2000 data and the 2003 data agree to better than $\pm 1 \mathrm{~km} \mathrm{~s}^{-1}$. Since these results were obtained using independent calibration data, they provide a clear indication that systematic errors in velocity differences between remote regions are not present.

Conversion of count rates to absolute values of surface brightness was obtained by comparison with calibrated data in Vannier et al. (2001). Thus the count rate of 2.15 counts pixel ${ }^{-1} \mathrm{~s}^{-1}$, at the peak of emission to the SE of TCC0016, corresponds to a velocity integrated brightness of $3.0 \pm 0.15 \times 10^{-5} \mathrm{~W} \mathrm{~m}^{-2} \mathrm{sr}^{-1}$.

The composite field observed within OMC1, shown in Fig. 1, is $90^{\prime \prime} \times 70^{\prime \prime}$. This allows us to address statistical issues involving scales ranging from 70 to $3 \times 10^{4} \mathrm{AU}$ or $3.4 \times 10^{-4}$ to $0.15 \mathrm{pc}$.

Velocities are expressed as $v_{\text {lsr }}$ by assigning a mean velocity of all the data of $12 \pm 6 \mathrm{~km} \mathrm{~s}^{-1}$ in the local standard of rest (Chrysostomou et al. 1997; Salas et al. 1999; O'Dell 2001). Since we are essentially concerned only with the relative velocities of regions within $\mathrm{OMC} 1$, the uncertainty in the value of $v_{\mathrm{lsr}}$ is not material to our discussion.

\section{3. $\mathrm{H}_{2}$ emission and excitation in the inner region of OMC1}

There is a wealth of data for the dense star-forming region of OMC1, even if consideration is restricted to IR observations of $\mathrm{H}_{2}$ emission. Reference to these extensive data, covering features such as the well-known "bullets" and "fingers" of $\mathrm{H}_{2}$ emission, and very high spatial resolution observations of $\mathrm{H}_{2}$ performed using the HST, VLT and CFHT may be found in recent work e.g. McCaughrean \& Mac Low (1997); Stolovy et al. (1998); Schultz et al. (1999); Lee \& Burton (2000); O'Dell (2001); Davis et al. (2001); Vannier et al. (2001); Doi et al. (2002); Gustafsson et al. (2003) (Paper I); Lacombe et al. (2004). Our account here is restricted to data specifically concerned with radial motion measured in Orion using $\mathrm{H}_{2}$ IR emission as a tracer (Sugai et al. 1995; Chrysostomou et al. 1997; Salas et al. 1999). Excitation mechanisms of $\mathrm{H}_{2}$ are however briefly considered here since these have a bearing on later discussion.

The high brightness of $\mathrm{H}_{2}$ emission in the $v=1-0 \mathrm{~S}(1)$ line, frequently exceeding $10^{-5} \mathrm{~W} \mathrm{~m}^{-2} \mathrm{sr}^{-1}$, indicates that the gas is of high column density. Since brightly emitting clumps are typically $1^{\prime \prime}$ to $2^{\prime \prime}$ in angular size $\left(1^{\prime \prime}=2.2 \times 10^{-3} \mathrm{pc}\right)$, the inference is that number densities are also high. The most feasible general mechanism of $\mathrm{H}_{2}$ excitation, for high brightness, is through C-type (magnetic) shocks (Smith \& Brand 1990; Kaufman \& Neufeld 1996a,b; Timmermann 1998; Wilgenbus et al. 2000; Vannier et al. 2001; Le Bourlot et al. 2002; Flower et al. 2003; Kristensen et al. 2003, 2005). Low velocity shock excitation in dense gas in regions of OMC1 shown in Fig. 1, has been analysed in detail for example in Vannier et al. (2001) and in Kristensen et al. (2003). Densities in the post-shock gas 
exceed $10^{7} \mathrm{~cm}^{-3}$. In addition, the data of Paper I graphically illustrate the presence of shocks through the clear spatial association of bright $\mathrm{H}_{2}$ emission with gas flows.

In addition to shocks, photon induced excitation of $\mathrm{H}_{2}$, in so-called photon dominated regions (PDRs), can play a role in yielding the observed $\mathrm{H}_{2}$ IR emission. This has been discussed in detail in Kristensen et al. (2003) who show that PDRs may contribute significantly in specific regions, for example in the SE (Peak 2) region, especially at the edge of clumps of material in this region. In strongly emitting zones, PDRs cannot yield sufficient brightness, even including effects of advection (Lemaire et al. 1999), high density, and the high UV flux from $\Theta^{1}$ Ori-C, in excess of $10^{5}$ times the standard UV field (Störzer $\&$ Hollenbach 1999).

\section{Statistical analysis}

\subsection{Filtering of data}

The presence of large scale velocity differences in OMC1 has been known for many years. Sugai et al. (1995), Chrysostomou et al. (1997) and Salas et al. (1999) have all used FP interferometry in OMC1 in the IR and studied the large scale velocity structure of the hot $\mathrm{H}_{2}$ component. From our observations (Paper I) we find that the gas in Peak 1 (NW of BN) is on average $10 \mathrm{~km} \mathrm{~s}^{-1}$ more blueshifted than in Peak 2 (SE of BN: Fig. 1; see also Fig. 2 in Paper I). This velocity difference has generally been viewed in the context of an outflow mechanism from the IRc2-complex and has previously been explained by an expanding shell (Scoville et al. 1982; Sugai et al. 1995), a bipolar outflow (Chrysostomou et al. 1997) or a spherical wind (Salas et al. 1999). The velocity difference could however be a manifestation of turbulent energy injected into the system and stirring of the gas in large scale vortices. Thus the nature of the velocity difference remains uncertain and in the following we will explore this question further.

As discussed in Miesch \& Bally (1994), Miesch \& Scalo (1995) and Miesch et al. (1999), large scale trends - such as a general velocity gradient - could dominate any statistical quantifier and give misleading results. This is due to the fact that the statistical methods lose spatial information and therefore depend on an absence of systematic trends within the data. It may therefore be argued that any large scale trends should be removed and only local velocity fluctuations studied. Ossenkopf \& Mac Low (2002) however point out that large scale systematic motions may be part of the turbulent cascade if these inject energy into the system and should therefore only be removed if the turbulence studied is exclusively driven on smaller scales.

In this connection, observations of molecular clouds seem to indicate that interstellar turbulence is driven on scales larger than the clouds themselves, and arguments have been presented that supernovae explosions dominate (Mac Low \& Klessen 2004). However, contributions to the continuous driving of the turbulence may also arise from protostellar outflows (Matzner \& McKee 2000) and stellar winds from massive O stars (Mac Low \& Klessen 2004). OMC1 is itself a region of highly active star formation. Observations ranging from X-ray (Garmire et al. 2000; Feigelson et al. 2002) to radio wavelengths (e.g. Churchwell et al. 1987; Zapata et al. 2004) reveal the presence of protostars, outflows (e.g. Genzel \& Stutzki 1989), HH objects (e.g. O'Dell et al. 1997; Doi et al. 2004) and mainline $\mathrm{OH}, \mathrm{H}_{2} \mathrm{O}$ and $\mathrm{SiO}$ maser emission (e.g. Norris 1984; Menten \& Reid 1995; Gaume et al. 1998; Greenhill et al. 2004b), noting that maser emission of the nature observed in $\mathrm{OMC} 1$ is associated with the presence of $\mathrm{O}$ stars. Thus, in OMC1 the turbulence could be driven locally by the powerful outflow from the BN-IRc2 complex, which contains a number of possible candidates such as source I, source $\mathrm{n}$ and BN itself (Menten \& Reid 1995; Gezari et al. 1998; Doeleman et al. 1999; Greenhill et al. 2004a; Shuping et al. 2004). Turbulence may also be driven on smaller scales by low mass star formation.

The alternative mechanism remains, as mentioned earlier, that the larger scale dynamics is predominantly part of a turbulent cascade driven on greater scales. For this reason we test the statistical quantifiers explored in this paper with the full velocity field and also the residual velocity fluctuations resulting from removing the large scale velocity trends.

The procedure of removal of large scale gradients, or filtering, follows Miesch \& Bally (1994). The removal is performed by convolving the data with a smoothing function and then subtracting the smoothed image from the original to obtain the residual image of the small scale velocity fluctuations. The residual image should then reveal any small scale structures that were superposed on the large scale trends in the original. The filtering or smoothing function used here is a twodimensional equally weighted moving average in the form of a square box. The optimal size of the filter is the broadest possible that removes the large scale gradient but leaves all other features. The 2D autocorrelation function of a velocity map can be used for detecting velocity gradients. These show up as anticorrelations in the direction of the gradient and recorrelations in the direction at right angles (Spicker \& Feitzinger 1988).

In the autocorrelation function of our velocity map the correlation persists in the NE-SW direction and decorrelation occur in the NW-SE direction. This indicates a large scale velocity gradient in the NW-SE direction which is consistent with the velocity difference between Peak 1 and 2 . The widest filter where no anticorrelated sidelobes are discernible in the autocorrelation function of the residual image is the optimal. By calculating the autocorrelation function for filters of varying size, the optimal filter size was found to be $14^{\prime \prime} \times 14^{\prime \prime}$ $(6400 \times 6400 \mathrm{AU})$. All further analysis has been carried out on both the original full velocity field and on the residual image obtained from filtering with the optimal filter. This latter will be referred to as the filtered velocity image or field. We find that it is certainly unwise to remove apparent large scale velocity trends and that the filtered image is not representative of the dynamics in $\mathrm{OMC} 1$, as discussed immediately below in Sect. 4.2.

\subsection{Size-linewidth relation}

Larson (1981) identified an empirical relation between linewidth (or velocity dispersion) and size for molecular clouds 
and clumps within clouds over scales of $\sim 0.1-100$ pc. Larson obtained a power law

$\Delta v_{\text {obs }} \propto R^{\alpha} \quad \alpha \simeq 0.38$

where $\Delta v_{\text {obs }}$ is the observed linewidth and $R$ is the effective radius of the object. This relationship essentially supports a model in which larger motions are associated with larger scales. More recent studies give a range of values for $\alpha$ over dimensions of 0.02-100 pc (e.g. Caselli \& Myers 1995; Peng et al. 1998) showing $\alpha$ varying between 0.2 and 0.7 . Some studies indicate that massive star forming regions tend toward the lower values (e.g. Caselli \& Myers 1995). There are however indications that the relation in Eq. (2) breaks down in the most massive star forming regions (Plume et al. 1997). A comprehensive overview was given in Goodman et al. (1998). Sizelinewidth measurements require that objects are well defined within a map and that a linewidth and size can be objectively assigned to objects within the field of observation. The definition of objects is obvious for isolated clouds, but encounters a certain degree of arbitrariness when dealing with clumps in a cloud.

The structure of OMC1, measured in vibrationally excited $\mathrm{H}_{2}$, reveals a very clumpy environment where the regions of bright emission are in many circumstances imposed on extended weaker emission. OMC1 is thus a case study in the difficulty of determining the physical extent of individual clumps in a turbulent environment. In order to circumvent this problem in computing a size-linewidth relation we follow the method developed by Ossenkopf \& Mac Low (2002). We remind the reader at this stage that our observations of linewidth are indirect. That is, we obtain the velocity associated with any pixel from the peak of the line profile observed with the low resolution Fabry-Perot, see Sect. 2. Thus the linewidth associated with any chosen assembly of pixels is given by the velocity dispersion of these peak velocities. This is in contrast to the standard technique in radio observations in which the velocity resolution of observations is a fraction of the linewidth, and the linewidth may then be obtained directly from the measured lineshape. The technique which we use here, that of taking the peak of the line for each pixel, was also used by Ossenkopf \& Mac Low (2002) (using centroid velocities). For this reason we refer below to the Larson relationship as a size-velocity dispersion relation, rather than the more familiar size-linewidth. In the present case we note that $\mathrm{H}_{2}$ emission is optically thin and there are no optical depth effects, such as those discussed in detail in Ossenkopf \& Mac Low (2002).

The average brightness weighted velocity dispersion, $\Delta v_{\mathrm{obs}}$, within regions of varying size, is estimated following the prescription of Ossenkopf \& Mac Low (2002). We compute the brightness weighted probability distribution function of the peak velocities in a circular region with a given radius and find the corresponding velocity dispersion from a fit to a Gaussian. This is repeated throughout the map and for a number of radii, $R$ (see Eq. (2)). For each radius the average velocity dispersion is calculated using intensity weighting. The relation between velocity dispersion and size is shown in Fig. 2 for both the full velocity image (upper frame) and the filtered velocity image (centre frame). Errors shown are the statistical
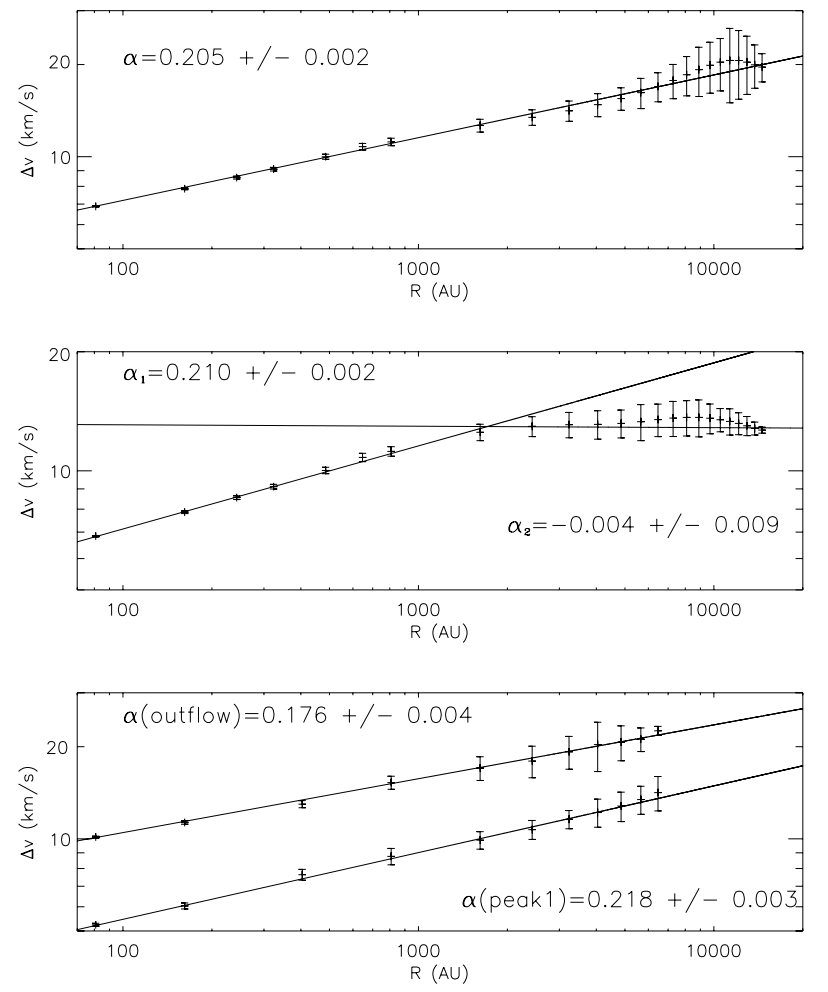

Fig. 2. Larson size-linewidth relations, Eq. (2). Top: velocity dispersion as a function of size for the full velocity field. A power law fit of index 0.205 is overlaid. Centre: velocity dispersion for the filtered velocity image. Power laws of index 0.210 and -0.004 are overlaid. Bottom: velocity dispersion for the outflow region (upper line) and Peak 1 (lower line) with power law fits overlaid.

standard error on the mean. Within the errors the relation for the full velocity field is consistent with a single power law over more than two orders of magnitude in $R$ with an exponent $\alpha=0.205 \pm 0.002$, although there also appears to be some deviation above $8000 \mathrm{AU}$. The value of the exponent agrees with the average value of $0.21 \pm 0.03$ that Caselli \& Myers (1995) found for massive cores in the Orion A and B clouds at scales $0.03-1 \mathrm{pc}$. It is striking that the Larson relationship appears both to hold in this very different regime and to have a very similar exponent as for massive cores, although we are dealing here with highly excited material.

The appearance of the region south-west of $\mathrm{BN}$ with fewer and more isolated clumps of emission, suggests that the dominating physical processes in this region are different from those in Peaks 1 and 2. In Peaks 1 and 2 the emission is more homogeneous and more spatially concentrated. All clumps with measurable radial velocities south-west of BN are blue-shifted. Nissen et al. (2005) provide clear evidence that these objects form the IR counterpart of an outflow detected in radio observations of $\mathrm{SiO}$ masers associated with a buried O-star within OMC1 (Menten \& Reid 1995; Doeleman et al. 1999; Greenhill et al. 2004a,b; Shuping et al. 2004). For this reason we refer to this zone as the "outflow region".

We have computed the size-velocity dispersion relation for the outflow region and Peaks 1 and 2 separately, in each case 
for the full velocity field (that is, without filtering of the data see Sect. 4.1). The resulting size-velocity dispersion relationships, shown for the outflow region and Peak 1, with Peak 2 omitted for clarity, are shown in the third frame of Fig. 2. This illustrates that the structure adheres to the Larson relationship in the outflow region south-west of BN but with a significantly lower slope and a somewhat higher proportionality parameter $\kappa$ in $\Delta v_{\text {obs }}=\kappa R^{\alpha}$ than for Peaks 1 and 2. In the latter two regions $\kappa$ 's and the exponents $\alpha$ are the same within observational error. The lower value of $\alpha$ associated with the outflow region supports the conclusion of Caselli \& Myers (1995) that lower exponents are characteristic of massive star-forming regions.

The relation based on the filtered velocity image in the centre frame of Fig. 2 shows that the velocity dispersion is not well represented by a single power law. At radii smaller than $\sim 1600$ AU the relation follows a power law of index 0.210 , essentially the same as for the full velocity field. This similarity of behaviour is expected since the filter applied to remove the velocity gradient has a width of 14" $~ 6400$ AU (Sect. 4.1), and an equivalent radius of $3200 \mathrm{AU}$, and thus should not affect smaller scales. In contrast to the full velocity field, the velocity dispersion for the filtered data at larger scales is constant within observational error as a function of size, in marked contradiction to the accepted form of the Larson relationship, with an index at scales larger than $1600 \mathrm{AU}$ of $-0.004 \pm 0.009$. In a turbulent medium, where the velocity distribution is expected to get broader with increasing size of the region, this is unnatural and can only be caused if some of the turbulent velocities have been artificially removed. We conclude that by removing the large scale gradient we have removed some of the turbulent energy. It follows therefore that the large scale motions in OMC1, reported both here and by a number of other authors (Scoville et al. 1982; Sugai et al. 1995; Chrysostomou et al. 1997; Salas et al. 1999) should be seen as representing real scales of a turbulent cascade. By implication the turbulence is driven at large scales and this suggests that turbulence on the scale of the map could be either the result of an energy cascade from still greater scales or injection of turbulent energy by the large scale outflows associated with massive star formation in the IRc2 region.

This in turn implies that the turbulence in the OMC1 region does not have a strong injection of energy at scales of less than $0.1 \mathrm{pc}$ and is thus not primarily driven on small scales, for example by low mass protostellar outflows. In support of this, the energy injected by a high mass stellar outflow considerably exceeds that of the sum total of low mass outflows. Elsewhere we have estimated (Nissen et al. 2005) that the mass outflow rate is of the order of $>10^{-3} M_{\odot} / \mathrm{yr}$ in the blue-shifted outflow, SW of $\mathrm{BN}$, with an average velocity of $18 \mathrm{~km} \mathrm{~s}^{-1}$. Low mass protostellar outflow rates are typically three orders of magnitude lower (Richer et al. 2000), with a similar velocity. This simple argument on the basis of energetics supports our conclusion that the injection of energy at the $0.1 \mathrm{pc}$ scale of massive stars outweighs on the global scale the energy input from low mass stars. However, as we find in Sect. 5, locally as opposed to globally, the character of the turbulence is strongly affected by low mass protostellar outflows.
The velocity gradient, as identified above, should consequently not be removed prior to analysis. In the following we thus mainly focus on the full velocity field containing velocities on all scales, but for completeness we have also included analysis of the filtered velocity image.

\subsection{Peak velocity PDFs}

The probability distribution function (PDF) of velocities is defined in terms of 3D velocity components as the number of pixels at a certain velocity vs. velocity. Here we are restricted to a set of radial velocities and we estimate the PDF of these velocities sampled over the spatial region in the plane of the sky shown in Fig. 1. This corresponds to the PDF of centroid velocities used in Miesch \& Scalo (1995); Miesch et al. (1999); Ossenkopf \& Mac Low (2002). An alternative procedure is to use the line profile of an optically thin line as an estimate of the PDF along the line-of-sight (Falgarone \& Phillips 1990, 1991; Falgarone et al. 1994; Ossenkopf \& Mac Low 2002), taking advantage of the high velocity resolution of radio-observations. Ossenkopf \& Mac Low (2002) provides a comparison of the two methods.

The shape of the wings of the PDF is diagnostic of intermittency, in which, at random scales and/or time intervals, energy is dissipated as heat in the turbulent medium. At any scale the removal of energy must involve those elements of the medium with the most prevalent velocities. Thus it is the velocities at the centre of the distribution which becomes most depleted. Thus the velocity distribution loses its initial Gaussian form, with the centre depressed and the wings relatively enhanced. Increasing degrees of intermittency create a transition from Gaussian $\left(\propto \exp \left(-x^{2}\right)\right)$ to exponential $(\propto \exp (-x))$ wings in a PDF.

Gaussian PDFs may be found in studies of decaying supersonic turbulence (Ossenkopf \& Mac Low 2002) and incompressible turbulence (e.g. Batchelor 1956; Jayesh \& Warhaft 1991). Exponential PDFs can be found in the literature of simulations, e.g. from inelastic collisions of clouds (Ricotti \& Ferrara 2002) and interactions of shells (Chappell \& Scalo 2001). Further relevant theoretical studies involve the stretched exponential form $\left(\alpha \exp \left(-a|x|^{\beta}\right)\right)$ where $\beta$ is fractional (e.g. Frisch \& Sornette 1997; Eggers \& Wang 1998), where $\beta=2$ reproduces the Gaussian PDF and $\beta=1$ the exponential form. Fractional $\beta$ values can arise from random processes whose overall effect accumulate in a multiplicative manner (see Sect. 4.4). Other studies are concerned with wings of PDFs in the form of power laws for example arising from stellar winds or outflows (Silk 1995).

Using line profile data for CO from various isotopes Falgarone \& Phillips (1990, 1991), Falgarone et al. (1994); Ossenkopf \& Mac Low (2002) do not find simple Gaussian behaviour for most observations. However, Falgarone \& Phillips (1991) showed that most of the PDFs could be represented by two Gaussians, with the wing component being about 3 times broader than the core component. The PDFs in the present work cannot be so represented. Other work uses centroid velocities, corresponding to the peak velocities used here. For example, 

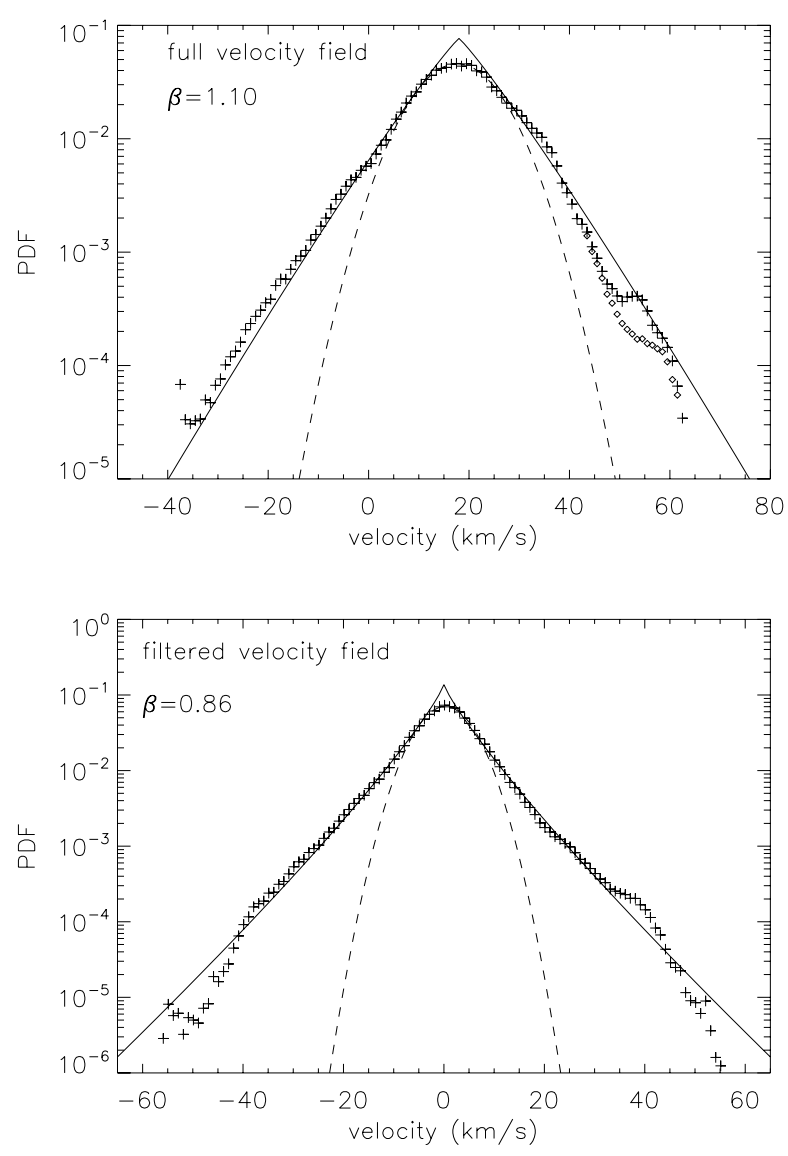

Fig. 3. Probability distribution functions of velocities. Top: (+) PDF for the full velocity field. $(\diamond)$ PDF with removal of velocities associated with the clump in Fig. 4. Bottom: PDF for the filtered data (see text). Stretched exponentials with $\beta=1.10$ and $\beta=0.86$ respectively are shown for comparison. The dashed lines are Gaussian fits to the core.

Miesch \& Scalo (1995), Miesch et al. (1999) found exponential tails in most of their PDFs, while Ossenkopf \& Mac Low (2002) found that the PDFs of the Polaris Flare could be reproduced by two Gaussians as in Falgarone \& Phillips (1991).

In the present work, the normalized PDF of peak velocities has been calculated by binning the velocities in intervals of $1 \mathrm{~km} \mathrm{~s}^{-1}$. The PDF can be estimated by assigning the same weight to every pixel or by weighting every velocity with the corresponding brightness in that pixel. By testing both methods we found that the chosen method of weighting does not influence the shape of the PDF significantly. Brightness weighting is less influenced by observational noise and is therefore used in the following analysis.

Figure 3 shows the peak velocity PDF of the full velocity field and of the filtered velocity image. The PDF of the filtered velocity image is artificially centered around $0 \mathrm{~km} \mathrm{~s}^{-1}$ through the action of filtering. The PDFs are shown on a log-linear scale where a Gaussian distribution would form a parabola and an exponential a straight line. To test the influence of uncertainties in the determination of velocities on the shape of the PDF, we have added to each velocity in the original dataset a random velocity from a Gaussian distribution with standard deviation corresponding to the uncertainty in the velocity of the pixel in question, as given by Eq. (1). We created a series of such velocity fields and calculated the PDF from each of them. The PDFs of velocity fields so generated are effectively indistinguishable from the PDF of the real data set, reflecting the fact that the large number of pixels used in the PDF, $\sim 3.2 \times 10^{6}$, reduces the effect of the observational noise to a negligible level. Again, since every bin of the histogram is populated by a large number of pixels, the relative error, $\sqrt{n} / n$, becomes very small, including taking account of the effective $3 \times 3$ pixels resolution. Except for the very least populated bins the statistical error bars would be smaller than the symbols in Fig. 3 and have therefore been omitted.

The PDFs in Fig. 3 are clearly not well represented by Gaussians except in the inner core, where Gaussians are shown as dashed lines in Fig. 3. Our data - for hot dense gas - show a different result to the data reported for much larger scales in Falgarone \& Phillips (1991) (for cool, diffuse gas and obtained using line profiles), where, as noted, a combination of two Gaussians fit the observations. The wings in the present work can be fitted by stretched exponentials (full lines). These lines are derived for the full velocity field data by fitting between +12 and $-32 \mathrm{~km} \mathrm{~s}^{-1}$ and yield $\beta=1.10 \pm 0.06$. A similar fit for the filtered data yields $\beta=0.86 \pm 0.05$.

It is clear from Fig. 3 that the PDFs are not symmetric and that the red wing displays large deviations from a smooth behaviour, especially at velocities of $50-60 \mathrm{~km} \mathrm{~s}^{-1}$, where a hump is seen in the PDF. By inspection of the velocity data we found that the hump results from a single structure in the field. This structure is the only clump of emission with velocities consistently larger than $50 \mathrm{~km} \mathrm{~s}^{-1}$ and is shown in Fig. 4 in $x y$-velocity space. If we ignore all pixels in this structure with velocities larger than $43 \mathrm{~km} \mathrm{~s}^{-1}$, then the PDF for the full field no longer contains a secondary hump at $55 \mathrm{~km} \mathrm{~s}^{-1}$ (diamonds in the upper frame in Fig. 3). This suggests that the structure involved is an independent entity which does not fit into the overall turbulent cascade. The nature of this dense fast moving object remains mysterious.

The shape of the PDF(s) in Fig. 3 can be further quantified by the brightness weighted statistical moments, computed directly from the dataset as:

mean $=\mu=\frac{\sum_{\text {map }} I(x, y) v(x, y)}{\sum_{\text {map }} I(x, y)}$

std. dev. $=\sigma=\sqrt{\frac{\sum_{\text {map }} I(x, y)[v(x, y)-\mu]^{2}}{\sum_{\text {map }} I(x, y)}}$

skewness $=\frac{\sum_{\text {map }} I(x, y)[v(x, y)-\mu]^{3}}{\sigma^{3} \sum_{\text {map }} I(x, y)}$

kurtosis $=\frac{\sum_{\text {map }} I(x, y)[v(x, y)-\mu]^{4}}{\sigma^{4} \sum_{\text {map }} I(x, y)}$

where the summations represented by map encompass all pixels in the data set.

The standard deviation quantifies the spread of the PDF, the skewness is a measure of the asymmetry and the kurtosis characterizes the deviation from a Gaussian profile. A Gaussian distribution has a kurtosis of 3 and larger values imply that the 


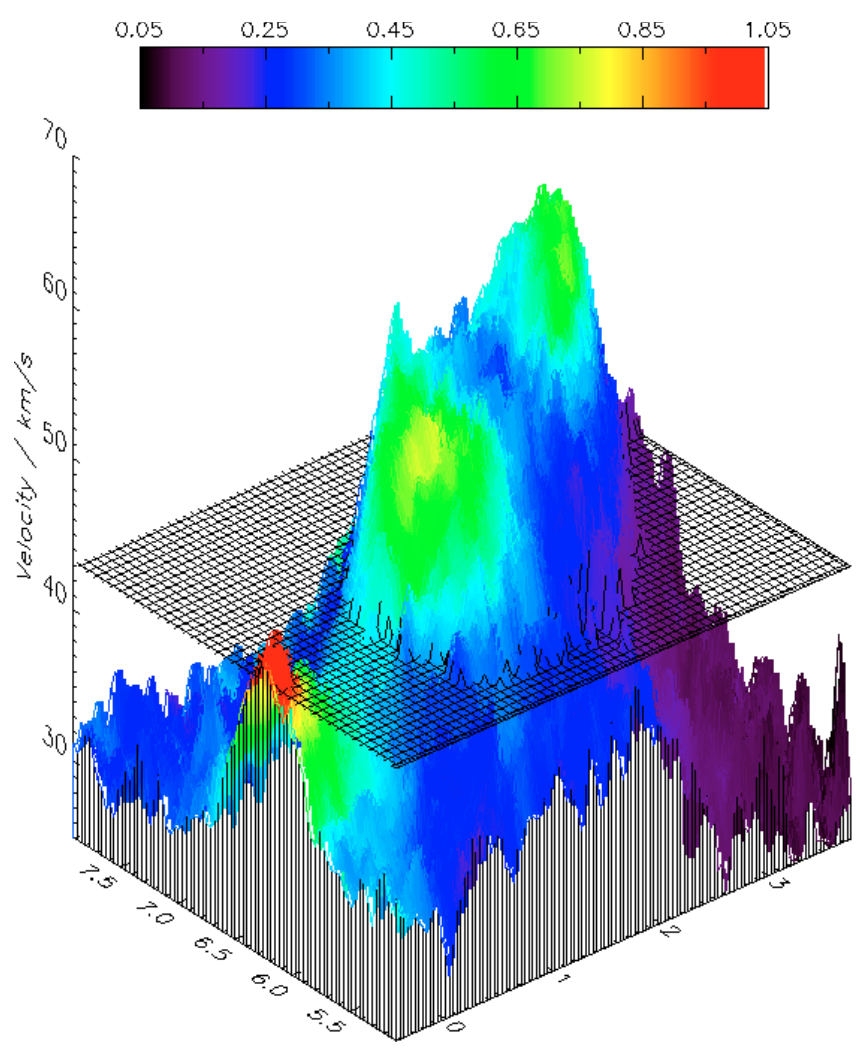

Fig. 4. The brightly emitting clump that causes the hump in the red wing of the PDF (Fig. 3, + symbols). The $x y$ plane is the plane of the sky, the vertical axis shows the radial velocity $v_{\mathrm{lsr}}$. The plane at $(x, y$, 43) shows the boundary of the region of pixels ignored in the PDF shown as $(\diamond)$ in Fig. 3. The centre of this clump is 6". 5 E, $1^{\prime \prime} 5 \mathrm{~N}$ of TCC0016. The colour scale refers to brightness in counts pixel ${ }^{-1} \mathrm{~s}^{-1}$, see Sect. 2

Table 1. Moments and stretching exponents of the peak velocity PDF for the full velocity field and the filtered velocity field

\begin{tabular}{ccccc}
\hline \hline Field & std-dev $/ \mathrm{km} \mathrm{s}^{-1}$ & skewness & kurtosis & $\beta$ \\
\hline full & 10.3 & -0.20 & 4.4 & 1.10 \\
filtered & 7.8 & -0.042 & 6.4 & 0.86 \\
\hline
\end{tabular}

PDF has relatively more prominent wings. An exponential distribution has a kurtosis of 6 . The calculated values for the PDF from our present data are listed in Table 1, showing a departure from Gaussian and from pure exponential behaviour. We conclude that the data show clear evidence of intermittency. There is also a skewness towards the blue. This may arise through preferential obscuration of red-shifted flows, compared to blueshifted, in this dusty and partly obscured region.

\subsection{Variance and kurtosis of velocity differences}

The PDFs presented above carry no spatial information. In order to retain some of the spatial characteristics of the velocity field and to quantify how velocities are spatially related within the medium, we now construct the probability distribution function of velocity differences between points separated by a certain distance in the plane of the sky, the lag. The resulting distributions should provide a more searching test of theoretical models than PDFs of peak or centroid velocities.

Velocity differences, $\Delta v=v(\boldsymbol{r})-v(\boldsymbol{r}-\boldsymbol{\tau})$, are used in this analysis. Here both $\boldsymbol{r}$ and $\boldsymbol{\tau}$, the lag, are two-dimensional space vectors. Previous authors, using both observational data and theoretical models, the latter for incompressible turbulence (Miesch \& Scalo 1995; Lis et al. 1998; Miesch et al. 1999) have described how the shape of the PDF changes with different lags. Specifically, PDFs were found to exhibit strong nonGaussian forms at small lags.

For homogeneous, isotropic turbulence - as assumed here - the same information can be obtained in a more compact form by investigating the structure functions as a function of lag magnitude, $L=|\tau|$. Structure functions of order $\mathrm{p}$ are defined as

$S_{\mathrm{p}}(L)=\left\langle|v(\boldsymbol{r})-v(\boldsymbol{r}-\boldsymbol{\tau})|^{p}\right\rangle=\left\langle|\Delta v|^{p}\right\rangle$.

This is in fact the traditional manner in which to characterise turbulence, as Kolmogorov originally prescribed (Kolmogorov 1941). In the inertial range of the turbulence the structure functions for Kolmogorov turbulence (non-dissipative, incompressible) obey power laws, $S_{\mathrm{p}}(L) \sim L^{\gamma(p)}$, and the exponents are called scaling exponents.

Here we study the second order structure function, $S_{2}(L)$, that is the variance, and the kurtosis, $S_{4}(L) / S_{2}(L)^{2}$ as a function of lag magnitude, $L$. In this manner information is obtained concerning the evolution of the PDF as a function of lag without the necessity for studying velocity difference PDFs directly. We have included a weighting function for the velocity differences, see Eqs. (8) and (9), and to avoid confusion with the traditional definition of the structure functions (Eq. (7)) we refer to Eq. (8) as the variance function and to Eq. (9) as the kurtosis function.

Both the variance and the kurtosis functions are powerful tools when comparing observations with different models of turbulence. For example, Kolmogorov turbulence predicts a power law variation of the variance function (see Eq. (8)) with lag magnitude, with a scaling exponent of $2 / 3$, whereas Miesch et al. (1999) find values between 0.33 and 1.05 (see below). Numerical and analytical studies of driven supersonic magnetohydrodynamic turbulence find a second order structure function exponent of 0.74 (Boldyrev et al. 2002).

Constructing the variance and kurtosis functions involves combining data from all pairs of pixels to obtain PDFs as a function of lag magnitude, $L=|\tau|$. The variance and kurtosis of the PDF of velocity differences are then

$$
\begin{aligned}
\sigma^{2}(L) & =\frac{\sum_{\text {map }} \sum_{|\tau|=L} w(\boldsymbol{r}) w(\boldsymbol{r}-\boldsymbol{\tau})(v(\boldsymbol{r})-v(\boldsymbol{r}-\boldsymbol{\tau}))^{2}}{\sum_{\text {map }} \sum_{|\tau|=L} w(\boldsymbol{r}) w(\boldsymbol{r}-\boldsymbol{\tau})} \\
K(L) & =\frac{\sum_{\text {map }} \sum_{|\boldsymbol{\tau}|=L} w(\boldsymbol{r}) w(\boldsymbol{r}-\boldsymbol{\tau})(v(\boldsymbol{r})-v(\boldsymbol{r}-\boldsymbol{\tau}))^{4}}{\sigma^{4}(L) \sum_{\text {map }} \sum_{|\tau|=L} w(\boldsymbol{r}) w(\boldsymbol{r}-\boldsymbol{\tau})}
\end{aligned}
$$

where the summations are performed over all pairs of pixels in the whole map that fulfill the requirement $|\tau|=L$. We have not used $L<4$ pixels $(0.15$ arcsec $)$, since this coincides with the spatial resolution of the data.

In the above, $\mathrm{w}$ is some weighting function. An important issue is whether the velocity data should be brightness 
weighted $(w(\boldsymbol{r})=I(\boldsymbol{r})$ in counts per second) or equally weighted $(w=1)$ in calculating the variance and kurtosis of the velocity difference PDFs. In order to address this problem, we need further to consider errors in the data. We have already removed data at the $2 \%$ level as described in Sect. 2. We now examine whether this cut-off is sufficiently low for the examination of variance and kurtosis functions. We find that a $2 \%$ cut-off is too low if we do not use intensity weighting but that it is acceptable if intensity weighting is included.

In order to investigate the effect of errors, we use equal weighting and calculate the variance function for a number of cut-off values in brightness. All the variance functions can be approximated by power laws, but we find that the scaling exponent, $\gamma$ in $\sigma^{2}(L) \propto L^{\gamma}$, increases with increasing cut-off value before converging to a constant value when the cut-off value reaches $\sim 9 \%$ of $I_{\max }$. This indicates that pixels with brightness lower than this value contribute significantly to the random noise and that they should be removed prior to analysis. This analysis emphasizes that caution should always be exercised when equal weighting is used since results may show dependence on the choice of cut-off.

The brightness weighted variance function is expected to be less influenced by noise. We find that the brightness weighted variance function including all pixels is essentially the same as the equally weighted variance function with a brightness cutoff of $9 \%$ of $I_{\max }$. Due to the lesser influence from noise we use the brightness weighted variance and kurtosis functions in the following.

Errors in the brightness weighted variance function resulting from uncertainties in the velocities have been calculated by using the error propagation law and taking the $1 \sigma$ uncertainty on the velocity in each pixel from Eq. (1). Due to the large number of pixel pairs that goes into the calculation of the variance function the relative error is typically $10^{-3}$ and therefore negligible.

The variance function is shown in Fig. 5 for the full velocity field in a log-log plot. The best fit of the variance function to a power law is found to have $\gamma=0.53 \pm 0.01$ and this is also shown in Fig. 5. For comparison, Miesch et al. (1999) obtained values of $\gamma$ between 0.33 and 1.05 for several molecular clouds at scales larger than $1.4 \times 10^{3} \mathrm{AU}$ while Ossenkopf \& Mac Low (2002) obtained $\gamma=0.94$ for the Polaris Flare at scales larger than $2000 \mathrm{AU}$. It is noteworthy that our value of $0.53 \pm 0.01$ is significantly lower than the Kolmogorov value of 0.67 . We also note that the variance function is closely related to the Larson relationship such that the exponent of the variance function should be of the order of twice the Larson exponent. This is approximately satisfied here.

The variance function in Fig. 5 in fact deviates significantly from a single power law, again underlining the nonKolmogorov nature of the gas dynamics. This is particularly apparent through a positive deviation around $800 \mathrm{AU}$ and a negative deviation around 100-200 AU. The curvature of the structure function may suggest the presence of a multi-fractal medium. At all events, several power laws operating in different ranges would appear necessary to fit the behaviour of the variance function.

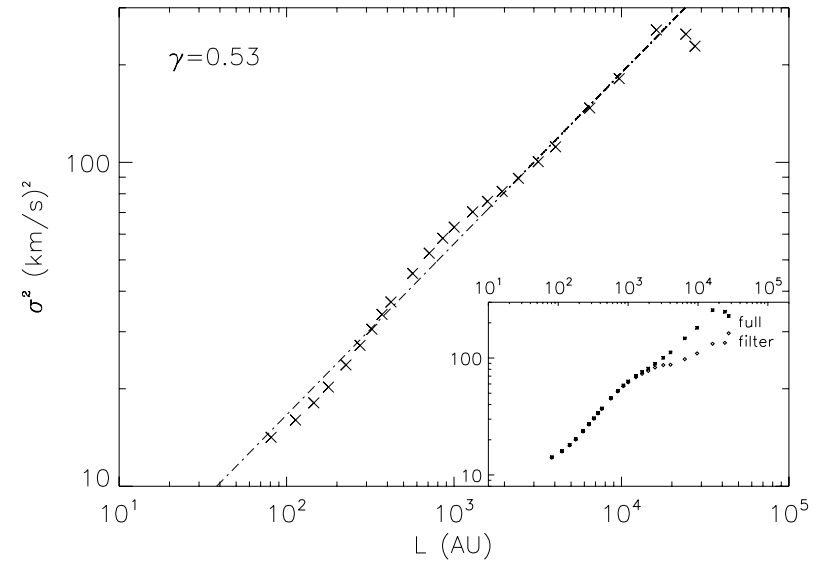

Fig. 5. The variance function for the full velocity field. A power law form with exponent 0.53 is overlaid. The inset displays the variance function of the filtered velocity image compared to the variance function of the full field.

The physical meaning attached to $\sigma^{2}(L)$ in Fig. 5 is that it approximately represents all energies in eddies below any scale $L$ (Davidson 2004). The deviation from a power law in Fig. 5 below 2000 AU therefore represents more energy at lower scales and implies that there is an excess of material in motion at $2000 \mathrm{AU}$ and below. This scale suggests that the excess arises from outflow events associated with star formation which result in energy injection at scales below $2000 \mathrm{AU}$. This feature is apparent from the data presented in Paper I and in Nissen et al. (2005). Conversely the material appears to suffer less velocity dispersion at scales below 300 AU. Thus material associated with these smaller scales appears to have dissipated some of its turbulent energy at larger scales. We speculate that these smaller less turbulent scales may be associated with protostellar nebulae or perhaps evaporating discs around protostars. In earlier work, purely on the basis of spatially resolved imaging (Vannier et al. 2001; Lacombe et al. 2004), a range of preferred scale sizes lying between $700 \mathrm{AU}$ and $1100 \mathrm{AU}$ was identified in Peak 2. In those cases and in the present case we suggest that the breaks in power laws are directly linked to scales associated with star formation.

The inset in Fig. 5 shows the effect of the removal of the large scale gradient on the variance function. The variance function of the filtered velocity image is identical to the variance function of the full field for scales smaller than half the size of the filter ( 3000 AU) above which the function flattens. It is clear that the filter has no effect on very small scales and that the removal of the large scale velocity structure causes the variance function to be systematically smaller on larger scales. This effect of the filtering has also been noted by Miesch et al. (1999) and is most likely an artefact due to the finite size of the map (Ossenkopf \& Mac Low 2002).

Turning now to the kurtosis function, the kurtosis is a measure of the degree of correlation in the motion at a certain scale relative to overall motions seen in the map. A velocity field with no spatial correlations would display a Gaussian shape. As mentioned above, a Gaussian has a kurtosis of 3. Thus values of kurtosis exceeding 3 indicate the strength of velocity 


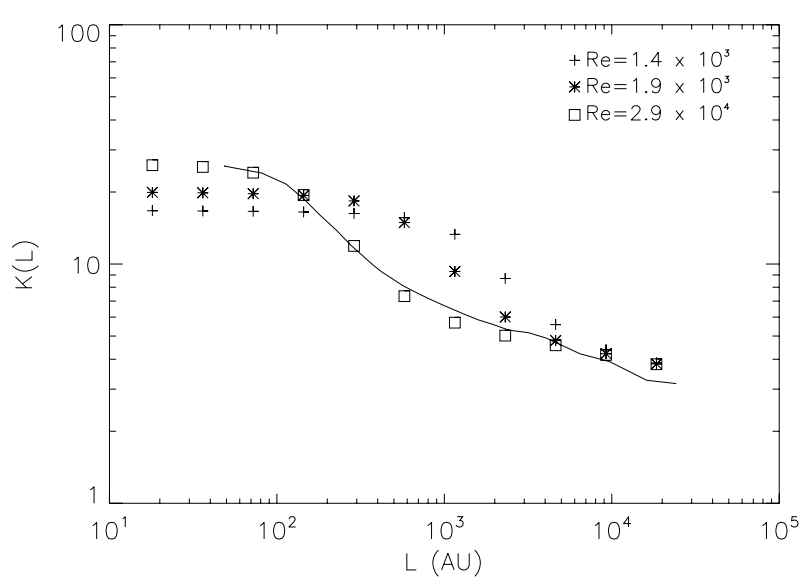

Fig. 6. The kurtosis function, Eq. (9), for the full velocity field (solid line). Theoretical predictions for 3 different Reynolds numbers from Eggers \& Wang (1998) are shown for comparison.

correlation at specific lags. From simulations Ossenkopf \& Mac Low (2002) found that kurtosis values above 3 can only be verified if the map has a scale considerably larger than the lag. As the lag approaches the size of the map, the value of the kurtosis tends to approach the Gaussian value of 3. This arises because the map cannot of course contain larger scales than the size of the map itself. Thus a kurtosis value around 3 is always expected at scales approaching the size of the map and provides no useful information on the velocity correlations at that scale.

Looking specifically at filamentary structure, Lis et al. (1998) found non-Gaussian wings of velocity difference PDFs at small scales evolving into Gaussians at larger scales, without quoting values of kurtosis. Miesch et al. (1999) found kurtosis values between 10 and 30 at the smallest lags $\left(1.4 \times 10^{3}-1.2 \times\right.$ $10^{4} \mathrm{AU}$ ), with the required Gaussian value of 3 at large lags of the order of the map size $\left(2 \times 10^{6} \mathrm{AU}\right)$. Similar behaviour was found by Ossenkopf \& Mac Low (2002). The kurtosis function derived from our present data is shown in Fig. 6 as a continuous line. The kurtosis values approach 30 at small lags (100 AU) and decrease gradually until reaching the value of 3 at large lags. The shape of the kurtosis function shows some similarity to those presented in Miesch et al. (1999). However a much more striking comparison may be found with results of a model of multifractal processes in Eggers \& Wang (1998). In Eggers \& Wang (1998) the kurtosis, called flatness in that work, is constant out to some lag beyond which the kurtosis decreases with increasing lag. The shape of the kurtosis was found by Eggers \& Wang (1998) to depend on the Reynolds number, where $R>1.1 \times 10^{4}$ marks a transition from a convex shape to a concave shape. The shape remains essentially unchanged for higher Reynolds numbers.

In order to compare with results in Eggers \& Wang (1998) we make an estimate of the Reynolds number of the flows in OMC1. The Reynolds number is given by $\operatorname{Re}=u l / v$, where $u$ and $l$ are the typical velocity and length scale, respectively, associated with the gas flows. The dynamical viscosity, $v$, can be approximated by $v \sim \lambda v_{\text {th }}$, where $\lambda$ is the mean-free-path of the particles and $v_{\text {th }}$ is the rms thermal velocity $\left(k T / \mu \mathrm{m}_{\mathrm{H}}\right)^{1 / 2}$ (Frisch 1995). Thus,

$\operatorname{Re}=\frac{u l}{\lambda v_{\mathrm{th}}}=u \ln \sigma \sqrt{\frac{\mu m_{\mathrm{H}}}{k T}}$

where the cross section, $\sigma$, for $\mathrm{H}_{2}-\mathrm{H}_{2}$ collisions is $2.7 \times$ $10^{-16} \mathrm{~cm}^{2}$ (Atkins 1990) and $n$ is the number density. The shocked regions observed here have a typical temperature of $2000-3000 \mathrm{~K}$ and gas densities are $10^{6}$ to $10^{7} \mathrm{~cm}^{-3}$. The velocity $u$ may be approximated by the velocity dispersion in our observed region and $u$ is therefore given by twice the standard deviation of the PDF of peak velocities (Table 1). The corresponding $l$ is the size of the region $\sim 3 \times 10^{4} \mathrm{AU}$. Using these values, we obtain a Reynolds number of $\operatorname{Re} \sim 3 \times 10^{9}$. The significance of this value is only that it is very large and greatly exceeds the critical value in Eggers \& Wang (1998) for which convex behaviour of the kurtosis function vs lag becomes concave. If we were to include magnetic effects, the magnetic Reynolds number might be substantially higher.

The Eggers \& Wang (1998) description of turbulent processes is ad hoc in the sense that it does not result from detailed high resolution three dimensional MHD simulations. Instead some reasonable assumptions are made concerning the cascade of turbulent energy, assuming it to be a stochastic process, isotropic and homogeneous in space, with certain additional properties which are briefly summarized below. Once one assumes that the turbulence is isotropic and homogeneous, the turbulence in the Fourier domain is represented by a spectrum which is a function of a single variable $r$, corresponding to a size of turbulent eddies. The approach of Eggers \& Wang (1998) is to impose a recipe for the cascade of energy from larger to smaller scales. The recipe states that energy at a given scale $r$ is transferred only to a scale $r / 2$. The amount of energy transferred, or more precisely the ratio $s$ of the velocity amplitudes between scale $r$ and $r / 2$ in equilibrium, is given by a probability distribution :

$p(s)=p \delta\left(s-s_{1}\right)+(1-p) \delta\left(s-s_{2}\right)$

with

$p=0.688, \quad s_{1}=0.699, \quad s_{2}=0.947$

where the probability $p$ and parameters $s_{1}$ and $s_{2}$ shown above were obtained by fitting to reproduce laboratory experimental data.

Equation (11) states that the probability $p$ is high $(\sim 2 / 3)$ for the ratio of velocities to be relatively small $\left(s=s_{1}\right)$, which corresponds to a low efficiency of energy cascade from one scale to the next. However there remains a probability of $\sim 1 / 3$ that there is efficient cascading, $s=s_{2}$. Starting from some chosen outer scale, this recipe allows for a very quick calculation of the energy at any given smaller scale by multiplying the probabilities for every cascade step lying between these two scales and producing the appropriate PDF semi-analytically. Hence the term multiplicative turbulence. The viscous cut-off of the turbulence is implemented through stopping the further cascading of energy if the Reynolds number at a given scale falls below a set value. The velocity fluctuation spectrum is replaced below that scale by an exponentially decaying tail. 
We show in Fig. 6 a comparison between our observed kurtosis and that presented in Eggers \& Wang (1998) for Reynolds numbers between $1.4 \times 10^{3}$ and $2.9 \times 10^{4}$, the highest which Eggers \& Wang (1998) show, noting once more that the form of the kurtosis function appears to be independent of Reynolds number above $\sim 10^{4}$. The lower Reynolds number cases are shown purely for comparison. The similarity of the kurtosis function between that for our data and that of Eggers \& Wang (1998) suggests that the model of a multifractal, multiplicative turbulent medium on which Eggers \& Wang (1998) is based may capture some of the physics of the turbulence in the hot component of the gas in OMC1.

\section{Analysis of individual clumps}

The high spatial resolution of the data allows the analysis of individual clumps of shocked gas with the same statistical methods as above. First, the dimensions of individual clumps must be defined and the following algorithm is used to identify the extent of any clump. The data are smoothed by a 9 by 9 pixel boxcar, in order that random fluctuations become unimportant. A clump is defined as a region which encompasses all pixels surrounding a local brightness maximum where the emission is situated on a continuously decreasing slope from the maximum brightness. Thus when we move in any direction starting from the pixel of maximum brightness and encounter a pixel with higher brightness than the preceding pixel, we define this as the boundary of the clump in this direction. As a further constraint we only use pixels with a brightness larger than $20 \%$ of the local brightness maximum. This avoids clumps becoming unrealistically large in the outer regions with low brightness and few brightness maxima. The $20 \%$ restriction has no effect in bright congested regions such as Peak 1 and 2. In passing, we note that we cannot rule out that some clumps so identified are the result of chance superpositions of two or more isolated clumps in the same line-of-sight. We have ignored this possibility.

With the above definition, we have delineated 170 clumps. These are in most part essentially the same features as analyzed in Nissen et al. (2005). The number of pixels in each clump ranges from 841 to 18026 , which corresponds to sizes of clumps between approximately 500 and $2200 \mathrm{AU}$. Due to the small size of the clumps, the earlier discussion concerning the removal of the large scale gradient is irrelevant here, since filtering does not affect the velocity field over such restricted regions.

\subsection{PDFs of clumps}

The PDFs of the 170 individual clumps have been calculated. The sensitivity of the shape of the PDF to uncertainties in the velocities has been investigated using simulations in the same way as in Sect. 4.3. Errors vary from clump to clump but do not influence the general shape of the PDF in any of the clumps. Eight representative PDFs with error estimates are shown in Fig. 7. Error bars shown represent the values spanned by the observed data and the simulated data. The PDFs for the clumps take on many different shapes. For most clumps the
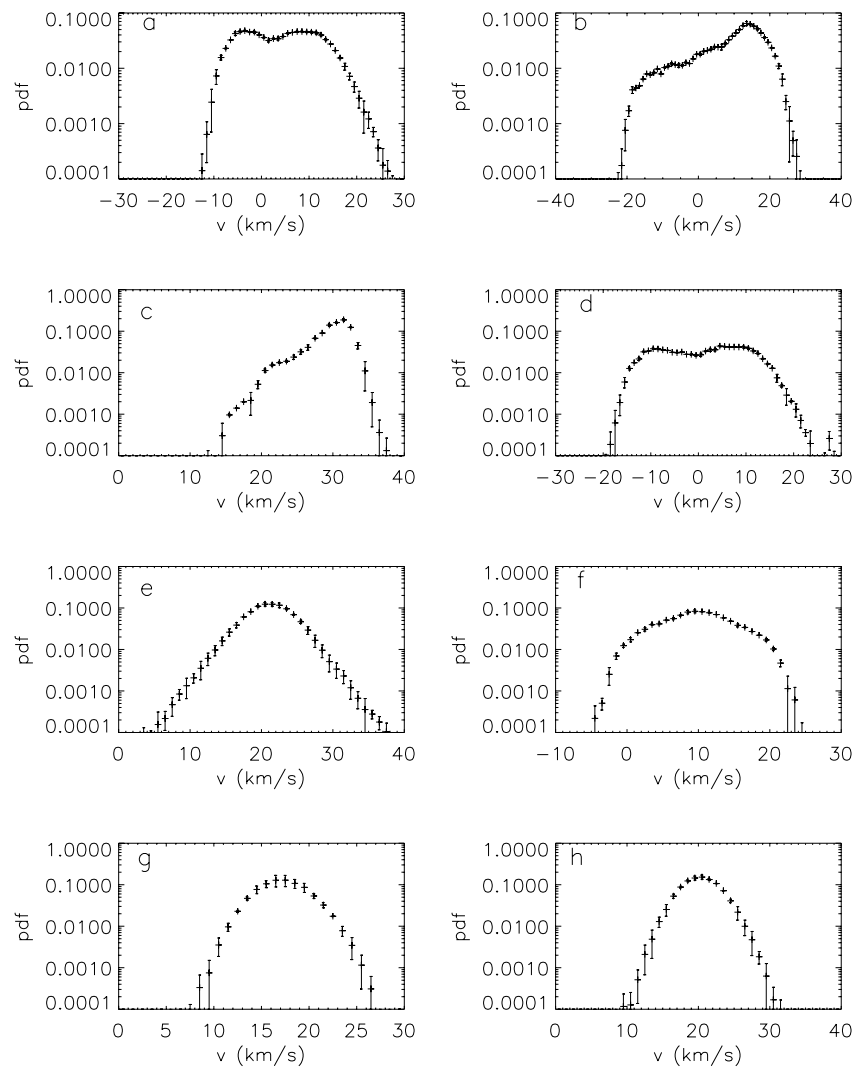

Fig. 7. PDFs of eight representative clumps. a), b), c) and d) are multimodal, e) and f) are stretched exponential, g) and h) are Gaussian. Positions are shown in Figs. 8, 10, 11.

shape is complex and appears bi- or multimodal. Bi- or multimodal PDFs, such as shown in Figs. 7a,b,c and d, can result from bipolar outflows from one or multiple protostellar objects. Most stars form in binary or multiple systems and the jets from these are found to be episodic or pulsed (Larson 2003, and references therein). 39 clumps have clear stretched exponential wings. Two such clumps are shown in Figs. 7e,f. 27 clumps have PDFs that can be fitted by a single Gaussian. Two examples are shown in Figs. $7 \mathrm{~g}, \mathrm{~h}$.

The spatial distribution of clumps with Gaussian PDFs, stretched exponential PDFs and bi- or multimodal PDFs is shown in Fig. 8. Clumps with Gaussian PDFs are found in all regions of the observed field, except in the central region around BN-IRc2, with essentially the same distribution as clumps with non-Gaussian PDFs. That is, there is no tendency for clumps with Gaussian PDFs, for example, to group together in small areas. Thus it seems that the turbulence dominating the BN-KL nebula can simultaneously produce both Gaussian and non-Gaussian PDFs in clumps with sizes of $\sim 10^{3} \mathrm{AU}$, and that the clumps with Gaussian and non-Gaussian PDFs are intermingled. However there appears to be some mechanism that inhibits the formation of clumps with Gaussian PDFs in the outflow region around BN-IRc2.

In connection with the above, Gaussian PDFs would of course arise if the velocity data had a significant noise contribution. However it turns out that this is not the case save perhaps in 3 of the 27 clumps mentioned above. For six of the 


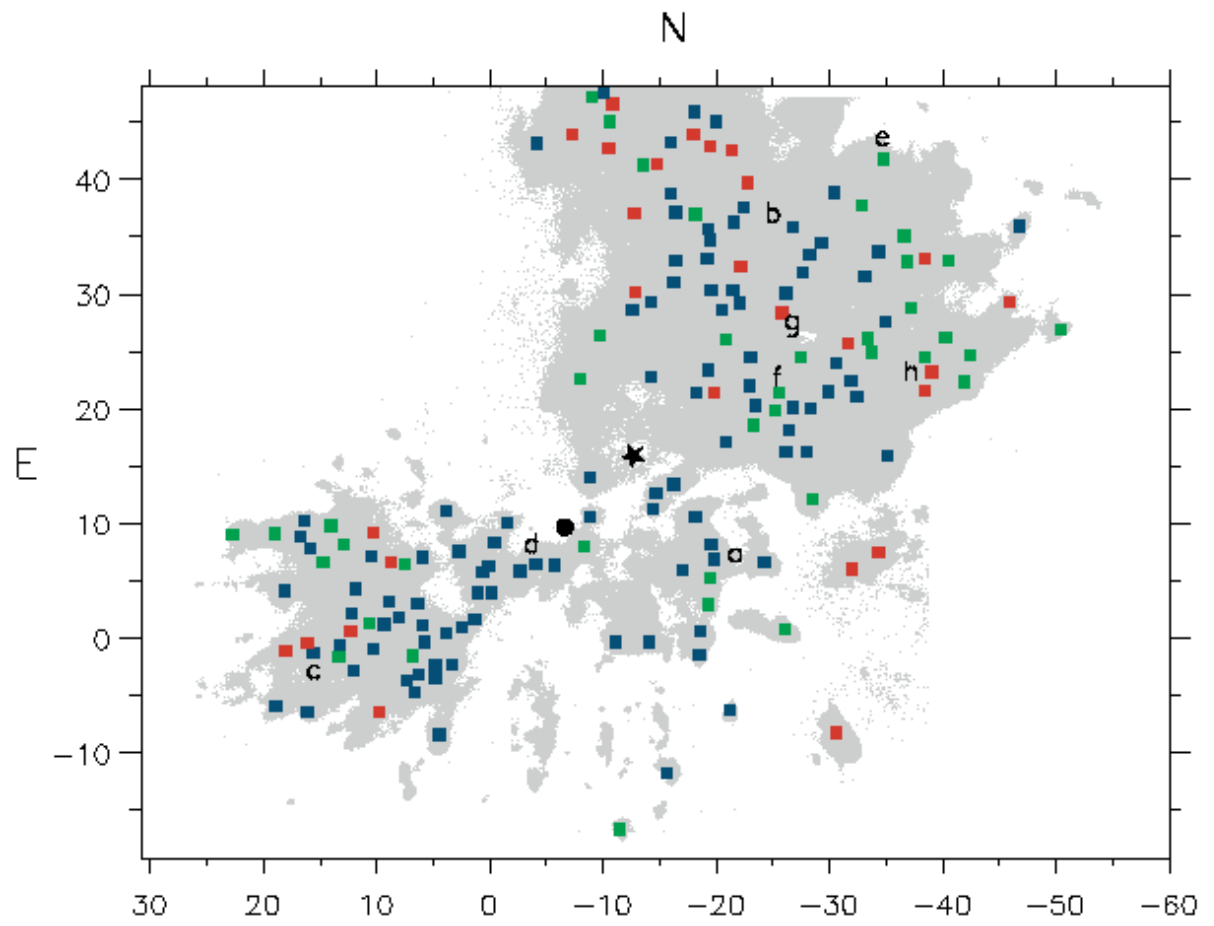

Fig. 8. Position of clumps with bi- or multimodal PDF (blue), stretched exponential PDF (green) and Gaussian PDF (red). The underlying grey background represents the spatial extent of velocity integrated $\mathrm{H}_{2}$ emission at $2.121 \mu \mathrm{m}$ with brightness greater than $\sim 2.4 \times 10^{-6} \mathrm{~W} \mathrm{~m}^{-2} \mathrm{sr}^{-1}$. The position of $\mathrm{BN}$ is marked with a star, IRc2 with a circle. a, b, c, d, e, f, g, h mark clumps shown in Figs. 7, 9.
27 clumps with Gaussian PDFs, the $1 \sigma$ uncertainty on the velocities (Eq. (1)) exceeds the half-width (one standard deviation) of the Gaussian PDF in $1 \%$ or more of the total number of pixels. The numbers of pixels are $1 \%, 4 \%, 5 \%, 28 \%, 32 \%$ and $54 \%$ of the totals. In the latter three of these six clumps the velocity distribution could be dominated by uncertainties in the velocities and these data may therefore be spurious, in the sense that the Gaussian character may be over-emphasized by noise.

\subsection{PDFs of velocity differences: variance functions for clumps}

The variance function of the velocity field has also been calculated for each clump, using Eq. (8). Most of the variance functions appear to be well approximated by power laws with a few exceptions, but the value of the scaling exponent $\gamma$ varies between essentially zero, that is, a flat distribution, and 1.61. The variance functions are shown in Fig. 9 for the eight representative clumps shown in Fig. 7.

The errors in the variance functions have been calculated in the same way as for the full region in Sect. 4.4. The magnitude of the errors depend on the brightness (see Eq. (1)) and the physical size of the clump, where size is the dominating parameter. Thus the smaller clumps have the larger errors, but even the smallest clumps have relative errors of no more than $10 \%$. Two of the clumps in Fig. 9 have relative errors of $10 \%$ which is of the same order as the size of the symbols in Fig. 9. Thus the errors have been omitted in Fig. 9. Errors in values of $\gamma$ arise from lack of precision of the power law fit, rather than from random errors in the variance. Typical errors in $\gamma$ are \pm 0.01 . Clumps with Gaussian PDFs show $\gamma=0.0-0.97$ including or excluding the 3 clumps with significant noise
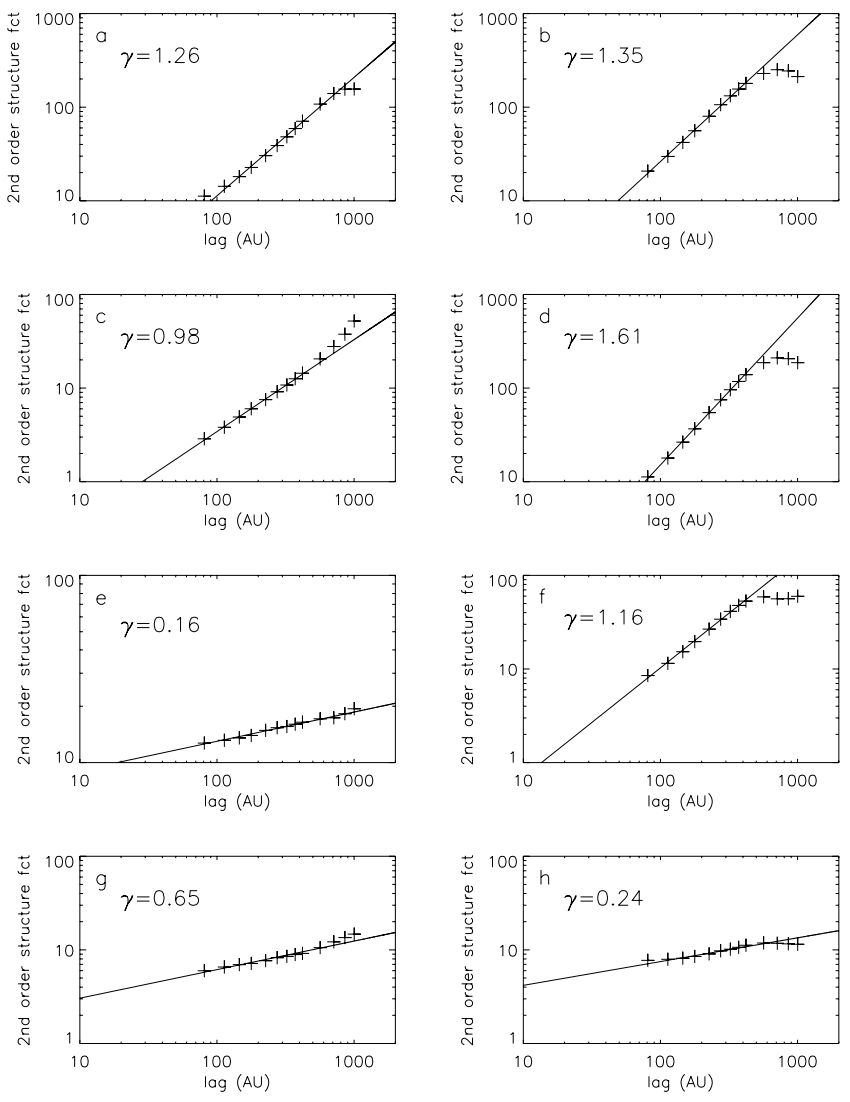

Fig. 9. The variance function for the eight individual clumps shown in Fig. 7. Power law fits are overlaid and the value of the exponent is given.

contributions (see Sect. 5.1). Clumps with non-Gaussian PDFs cover the whole range of $\gamma=0.0-1.61$. 


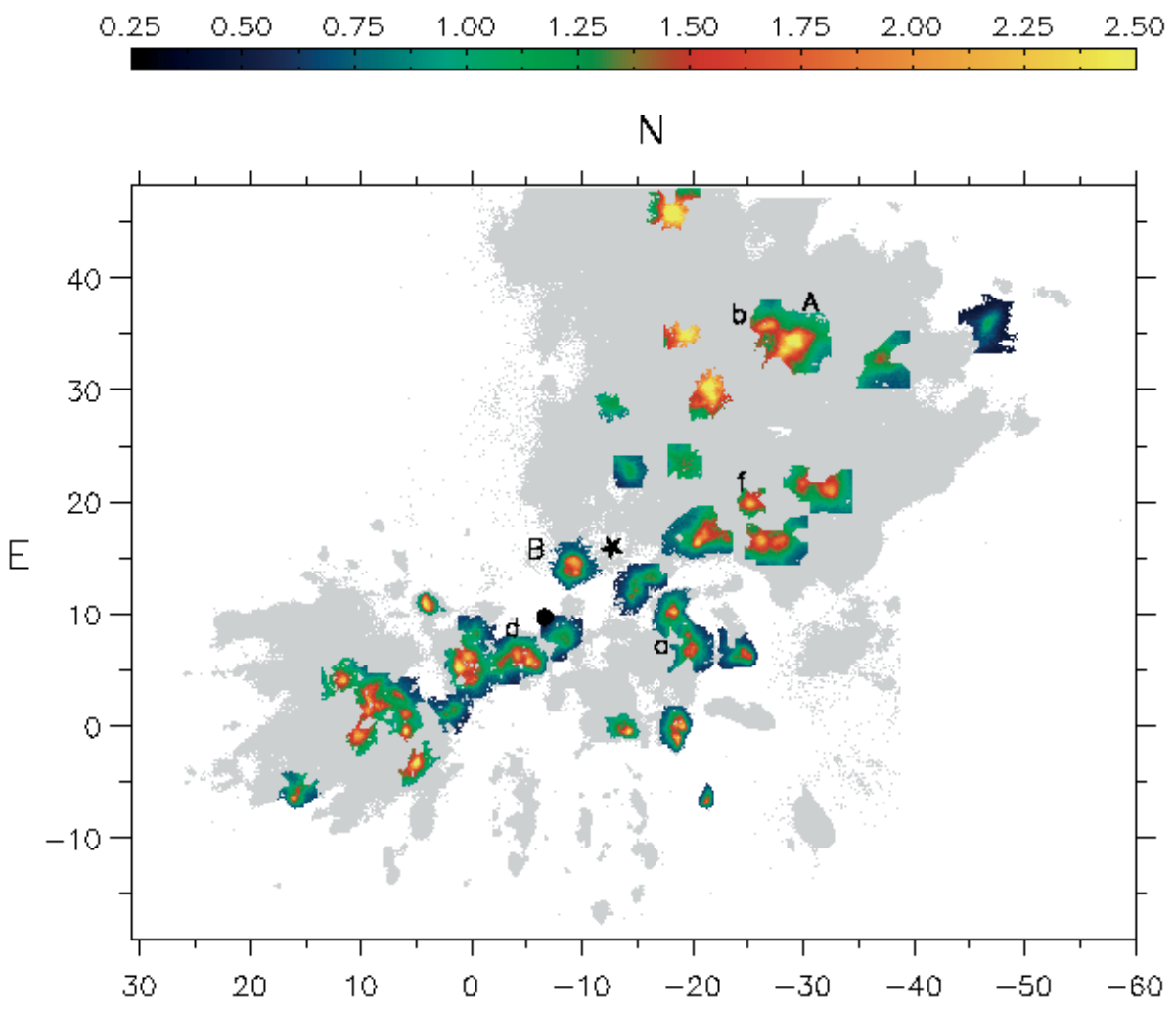

Fig. 10. Clumps with the variance function scaling exponent $\gamma>1.0$. As in Fig. 8, the underlying grey background represents the spatial extent of velocity integrated $\mathrm{H}_{2}$ emission at $2.121 \mu \mathrm{m}$ with brightness greater than $\sim 2.4 \times 10^{-6} \mathrm{~W} \mathrm{~m}^{-2} \mathrm{sr}^{-1}$. The position of $\mathrm{BN}$ is marked with a star, IRc2 with a circle. Colours represent brightness in counts per pixel per second in the clumps analyzed in Sect. 5. A and B mark sites of protostellar candidates. a, b, d, f mark clumps shown in Figs. 7, 9

A high value of $\gamma$ represents more ordered structure in the velocity field and large velocity gradients within the clump. This suggests the presence of ordered shock structure. Figure 10 shows the spatial distribution of clumps with $\gamma>1.0$, that is, clumps with the highest degree of order in the velocity field. The less ordered clumps with $\gamma<0.67$, the Kolmogorov value, are shown in Fig. 11. Examination of Figs. 10 and 11 shows that the value of $\gamma$ does not correlate with brightness, that is, high $\gamma$ can occur equally for strong and weak emission clumps. Furthermore, in Peaks 1 and 2 - but not in the central region around $\mathrm{BN}-\mathrm{IRc} 2$, see below - clumps with high $\gamma$ may be found mixed with clumps with low $\gamma$ in the same spatial region. A theory of star formation including turbulence, selfgravity, bipolar outflows, etc. should thus be able to reproduce such a range of exponents of the variance function within a limited physical region.

The majority of clumps with $\gamma$ less than the Kolmogorov value of 0.67 resides in Peaks 1 and 2 as seen in Figs. 10 and 11. The major part of these corresponds to clumps with measured velocities which display no clear structure and whose relative velocities within any clump do not exceed $5 \mathrm{~km} \mathrm{~s}^{-1}$ (Nissen et al. 2005), accordingly giving low values of the exponent. We suggest that some of these regions may arise from photodissociation regions (PDRs) involving an irregular surface containing a variety of lines of sight, illuminated by $\theta^{1}$ Ori-C, in a model essentially as described in Field et al. (1994) for the PDR NGC 2023. This would be consistent with a region lacking regular structure. However some low $\gamma$ clumps are very bright and cannot be reconciled with a PDR excitation model. These are likely to be shocks travelling across the line of sight.

Many clumps with high $\gamma$ (Fig. 10) are situated in the vicinity of the BN-IRc2 complex in the central region. Moving away from BN-IRc2, their occurrence becomes progressively less. Some of the high $\gamma$ clumps possess morphologies which resemble bow shocks (e.g. at $\left(-18^{\prime \prime}, 0^{\prime \prime}\right)$ and $\left(-21^{\prime \prime},-7^{\prime \prime}\right)$, see Fig. 10) and are part of the blue-shifted lobe of a bipolar outflow from radio source I, itself associated with a massive Ostar (Menten \& Reid 1995; Doeleman et al. 1999; Greenhill et al. 2004a,b; Shuping et al. 2004; Nissen et al. 2005). Other high $\gamma$ clumps correspond to protostellar candidates identified in Paper I and arise from outflows created internally in the clumps e.g. at $\left(-30^{\prime \prime}, 35^{\prime \prime}\right)$ and $\left(-10^{\prime \prime}, 15^{\prime \prime}\right)$, marked A and B in Fig. 10.

In contrast to regions of high $\gamma$, regions of low $\gamma$ are notably absent around BN-IRc2 and in the vicinity of source I, and tend to congregate where regions of high $\gamma$ are scarce. Our view of turbulent motion, based on models of turbulence, like that for example of Eggers \& Wang (1998), deals only with spatial scales and not directions. Thus we tend to consider only isotropic turbulence. However in the central region between Peaks 1 and 2, and also to the south-west of BN, the outflow from source I imposes a strong constraint on the radial motion of clumps in that region, where as mentioned clumps show a strong blue-shift. Motion is evidently not isotropic here. We suggest that high values of $\gamma$ arise in this region because effects of turbulence in the radial direction are effectively swamped in the variance function by large blue-shifted motions and the turbulence tends to $2 \mathrm{D}$ rather than isotropic $3 \mathrm{D}$. As we move away from the region of the blue-shifted outflow, low $\gamma$ clumps are once more encountered (see Fig. 11). It is an interesting challenge to theory to test if imposition of a strong outflow has the effects that we observe here on values of $\gamma$, as a check on our interpretation. Models should also demonstrate the absence of Gaussian PDFs. 


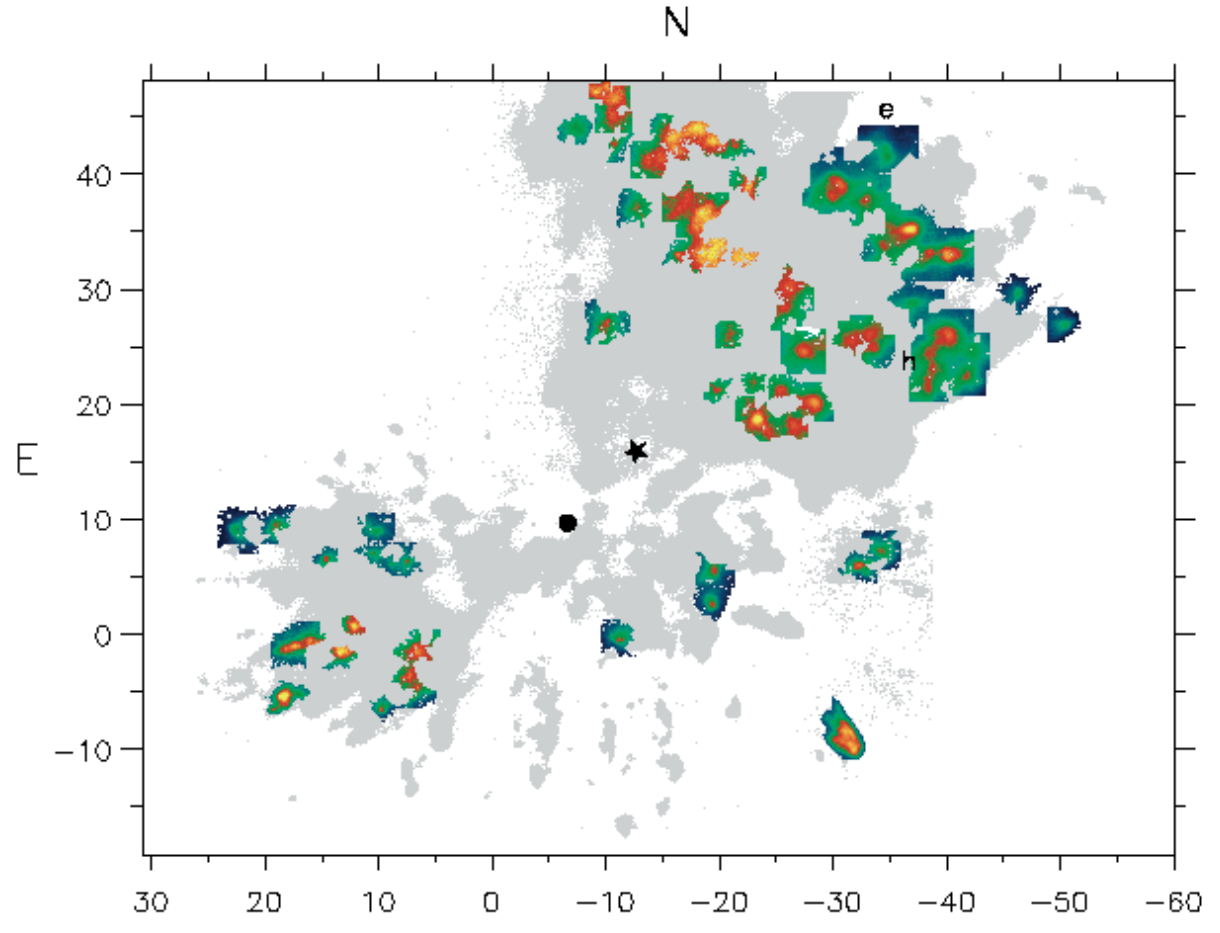

Fig. 11. Clumps with the variance function scaling exponent $\gamma<0.67$. Grey background as in Fig. 10. The position of $\mathrm{BN}$ is marked with a star, IRc2 with a circle. Colours represent brightness in counts per pixel per second in the clumps analyzed in Sect. 5. e, h mark clumps shown in Figs. 7, 9.
We now consider high $\gamma$ regions outside the blue-shifted outflow zone, that is, those which are well-mixed with regions of low $\gamma$. The formation and evolution of protostellar objects involve periods where a high degree of order in the velocity field surrounding the protostar is expected. A bipolar outflow encountering the circumstellar envelope would for example shock-excite the gas while creating an organized velocity field, with accompanying high $\gamma$. The detection of clumps with high values of $\gamma$ could therefore potentially allow an independent method of identifying early protostellar objects in the Class $0 / 1$ phase. This method may prove of value with the advent of very high spatial resolution radio maps with the Atacama Large Millimetre Array.

\section{Conclusions}

The fraction of gas studied in the present work is highly excited and very dense and quite distinct from gas whose statistical properties have been studied in earlier work. The latter refers to much larger scales and to much cooler, lower density and relatively quiescent gas. Nevertheless we have found that the hot dense gas in OMC1 nicely follows the general trends observed in earlier studies, down to the smallest, densest scales investigated here.

Our major conclusions from the statistical analysis of flows of $\mathrm{H}_{2}$ in OMC1 may be outlined as follows:

1. The size-linewidth relation of Larson (1981) is recovered at the small scales investigated here. The scaling exponent is $\alpha=0.205 \pm 0.002$, which agrees with the average value of $0.21 \pm 0.03$ that Caselli \& Myers (1995) found in Orion at scales 0.03-1 pc, using CO as an indicator (and which did not in fact include $\mathrm{OMC} 1$ ).

2. We find that the outflow, shown elsewhere (Nissen et al. 2005) to originate from a young and deeply buried O-star, between Peaks 1 and 2, generates structure which also follows the Larson relationship. Thus quite different environments preserve the Larson relationship. The velocity dispersion is however higher for any given size of object than elsewhere in the field and the exponent is significantly smaller than in the full region.

3. If we use velocity filtered data, removing the largest scales which appear as an apparent gradient of velocity, the Larson relationship breaks down. We conclude that the large scale motions are an inherent part of the turbulent cascade and should therefore not be removed from the data. Large scale injection of turbulent energy is the dominant process and outflows from massive star(s) in the IRc2 complex may contribute a substantial part of the driving of the turbulence at the scale of $0.1 \mathrm{pc}$.

4. The probability distribution function (PDF) of velocities is best fitted by an exponential or a weakly stretched exponential and departs strongly from Gaussian. This suggests that the turbulence in the region studied here is characterized by intermittency.

5. The variance function of the velocity differences is not well represented by a single power law. A multifractal model is implied. The best fit scaling component is $0.53 \pm 0.01$, significantly lower than the Kolmogorov value of 0.67 , underlining the non-Kolmogorov nature of the turbulence.

6. The behaviour of the variance function shows that there is a preferred scale size in the medium below $2000 \mathrm{AU}$ reflecting the presence of protostellar zones of this dimension and below. There is also clear evidence of less turbulent structure below $300 \mathrm{AU}$, perhaps representing a population of protostellar disks which have expelled part of their turbulent energy.

7. As further evidence of the multifractal nature of the medium, the kurtosis function of the velocity differences 
closely resembles that of the multifractal model of Eggers \& Wang (1998) for high Reynolds numbers.

8. Analysis of 170 individual clumps with sizes between 500 and $2200 \mathrm{AU}$ opens a window on a regime of scales that has never before been explored using statistical techniques. Studies at these scales reveal considerable diversity of velocity PDFs, with some Gaussian, some showing evidence of intermittency and many with complex structure, reflecting multiple outflows. Variance functions are approximately power laws, with exponents varying between zero and 1.61. There is no spatial association between high and low exponent clumps nor is there any association between Gaussian PDFs and high or low exponents.

9. To emphasize the last point, clumps with a variety of forms of the PDF and different values of the scaling exponent of the variance function are found in the same spatial region and in the whole region covered by the observations.

10. An outflow region associated with a deeply embedded O-star between Peaks 1 and 2 shows markedly different statistical characteristics from Peaks 1 and 2. Clumps in the vicinity of the BN-IRc2 complex typically show high scaling exponents in the variance function. This may be due to the action of the energetic outflow imposed on intrinsic turbulent motion within individual clumps. At all events high and low exponent clumps are spatially segregated in this zone, with low exponent clumps found only at the edges.

11. The outflow region apart, our results suggest that analysis using variance functions could be a useful way in which to establish the presence of early star-forming regions.

The above results constitute a challenge for numerical simulations of turbulence in star forming regions. To our knowledge the resolution of any present numerical simulation is substantially lower than the resolution of these observations, but advances in computer technology will soon allow simulations to reach the scales encountered here. A self-consistent theory of star formation including self-gravity and MHD turbulence should be able to reproduce the features outlined in this paper. Thus the size-linewidth relation, the probability distribution function of peak velocities and the variance and the kurtosis of velocity differences as a function of lag form a dataset which models of star-forming regions should aim to reproduce.

Acknowledgements. The authors would like to thank Dr. Wang for supplying us with numerical values for inclusion in Fig. 6. D.F. and M.G. would like to acknowledge the support of the Aarhus Centre for Atomic Physics (ACAP), funded by the Danish Basic Research Foundation and the Instrument Centre for Danish Astrophysics (IDA), funded by the Danish Natural Science Research Council. DF would also like to thank the Observatoire Paris-Meudon for support during the period in which this work was performed. J.L.L. would like to acknowledge the support of the PCMI National Program funded by the CNRS. We would also like to thank the Directors and Staff of the CFHT for making possible the observations reported in this paper. We should also like to acknowledge the helpful comments made by the referee.

\section{References}

Atkins, P. 1990, Physical Chemistry (Oxford University Press) Bally, J., O’Dell, C. R., \& McCaughrean, M. J. 2000, AJ, 119, 2919
Batchelor, G. 1956, The Theory of Homogeneous Turbulence (Cambridge: Cambridge University Press)

Boldyrev, S., Nordlund, ̊̊., \& Padoan, P. 2002, ApJ, 573, 678

Caselli, P., \& Myers, P. C. 1995, ApJ, 446, 665

Chappell, D., \& Scalo, J. 2001, MNRAS, 325, 1

Chrysostomou, A., Burton, M. G., Axon, D. J., et al. 1997, MNRAS, 289, 605

Churchwell, E., Wood, D. O. S., Felli, M., \& Massi, M. 1987, ApJ, 321, 516

Clénet, Y., Le Coarer, E., Joncas, G., et al. 2002, PASP, 114, 563

Davidson, P. 2004, Turbulence. An introduction for scientist and engineers (Oxford University Press)

Davis, C. J., Ray, T. P., Desroches, L., \& Aspin, C. 2001, MNRAS, 326,524

Doeleman, S. S., Lonsdale, C. J., \& Pelkey, S. 1999, ApJ, 510, L55

Doi, T., O’Dell, C. R., \& Hartigan, P. 2002, AJ, 124, 445

Doi, T., O'Dell, C. R., \& Hartigan, P. 2004, AJ, 127, 3456

Eggers, J., \& Wang, Z. 1998, Phys. Rev. E, 57, 4281

Falgarone, E., Lis, D. C., Phillips, T. G., et al. 1994, ApJ, 436, 728

Falgarone, E., \& Phillips, T. G. 1990, ApJ, 359, 344

Falgarone, E., \& Phillips, T. G. 1991, in Fragmentation of Molecular Clouds and Star Formation, IAU Symp., 147, 119

Feigelson, E. D., Broos, P., Gaffney, J. A., et al. 2002, ApJ, 574, 258

Field, D., Gerin, M., Leach, S., et al. 1994, A\&A, 286, 909

Flower, D. R., Le Bourlot, J., Pineau des Forêts, G., \& Cabrit, S. 2003, MNRAS, 341, 70

Frisch, U. 1995, Turbulence. The legacy of A.N. Kolmogorov (Cambridge: Cambridge University Press)

Frisch, U., \& Sornette, D. 1997, J. Phys. I France, 7, 1155

Garmire, G., Feigelson, E. D., Broos, P., et al. 2000, AJ, 120, 1426

Gaume, R. A., Wilson, T. L., Vrba, F. J., Johnston, K. J., \& Schmid-Burgk, J. 1998, ApJ, 493, 940

Genzel, R., \& Stutzki, J. 1989, ARA\&A, 27, 41

Gezari, D. Y., Backman, D. E., \& Werner, M. W. 1998, ApJ, 509, 283

Goodman, A. A., Barranco, J. A., Wilner, D. J., \& Heyer, M. H. 1998, ApJ, 504, 223

Greenhill, L. J., Gezari, D. Y., Danchi, W. C., et al. 2004a, ApJ, 605, L57

Greenhill, L. J., Reid, M. J., Chandler, C. J., Diamond, P. J., \& Elitzur, M. 2004b, in IAU Symp., 221, 155

Gustafsson, M., Kristensen, L. E., Clénet, Y., et al. 2003, A\&A, 411, 437 (Paper I)

Jayesh, \& Warhaft, Z. 1991, Phys. Rev. Lett., 67, 3503

Kaufman, M. J., \& Neufeld, D. A. 1996a, ApJ, 456, 611

Kaufman, M. J., \& Neufeld, D. A. 1996b, ApJ, 456, 250

Kolmogorov, A. N. 1941, Dokl. Akad. Nauk, 30, 301

Kristensen, L. E., Gustafsson, M., Field, D., et al. 2003, A\&A, 512, 727

Kristensen, L. E., Ravkilde, T., Field, D., Lemaire, J. L., \& Pineau des Forêts 2005, in preparation

Lacombe, F., Gendron, E., Rouan, D., et al. 2004, A\&A, 417, L5

Larson, R. B. 1981, MNRAS, 194, 809

Larson, R. B. 2003, Rep. Prog. Phys., 66, 1651

Le Bourlot, J., Pineau des Forêts, G., Flower, D. R., \& Cabrit, S. 2002, MNRAS, 332, 985

Lee, J.-K., \& Burton, M. G. 2000, MNRAS, 315, 11

Lemaire, J. L., Field, D., Maillard, J. P., et al. 1999, A\&A, 349, 253

Lis, D. C., Keene, J., Li, Y., Phillips, T. G., \& Pety, J. 1998, ApJ, 504, 889

Mac Low, M., \& Klessen, R. S. 2004, Rev. Mod. Phys., 76, 125

Matzner, C. D., \& McKee, C. F. 2000, ApJ, 545, 364

McCaughrean, M. J., \& Mac Low, M. 1997, AJ, 113, 391 
Menten, K. M., \& Reid, M. J. 1995, ApJ, 445, L157

Miesch, M. S., \& Bally, J. 1994, ApJ, 429, 645

Miesch, M. S., Scalo, J., \& Bally, J. 1999, ApJ, 524, 895

Miesch, M. S., \& Scalo, J. M. 1995, ApJ, 450, L27

Nissen, H., Gustafsson, M., Field, D., \& Lemaire, J. 2005, in preparation

Nordlund, Å., \& Padoan, P. 2003, Lect. Notes Phys. (Berlin Springer Verlag), 614, 271

Norris, R. P. 1984, MNRAS, 207, 127

O'Dell, C. R. 2001, ARA\&A, 39, 99

O'Dell, C. R., Hartigan, P., Lane, W. M., et al. 1997, AJ, 114, 730

Ossenkopf, V., \& Mac Low, M.-M. 2002, A\&A, 390, 307

Peng, R., Langer, W. D., Velusamy, T., Kuiper, T. B. H., \& Levin, S. 1998, ApJ, 497, 842

Plume, R., Jaffe, D. T., Evans, N. J., Martin-Pintado, J., \& Gomez-Gonzalez, J. 1997, ApJ, 476, 730

Richer, J. S., Shepherd, D. S., Cabrit, S., Bachiller, R., \& Churchwell, E. 2000, Protostars and Planets IV, 867

Ricotti, M., \& Ferrara, A. 2002, MNRAS, 334, 684

Salas, L., Rosado, M., Cruz-González, I., et al. 1999, ApJ, 511, 822
Schultz, A. S. B., Colgan, S. W. J., Erickson, E. F., et al. 1999, ApJ, 511,282

Scoville, N. Z., Hall, D. N. B., Ridgway, S. T., \& Kleinmann, S. G. 1982, ApJ, 253, 136

Shuping, R. Y., Morris, M., \& Bally, J. 2004, AJ, 128, 363

Silk, J. 1995, ApJ, 438, L41

Smith, M. D., \& Brand, P. W. J. L. 1990, MNRAS, 242, 495

Spicker, J., \& Feitzinger, J. V. 1988, A\&A, 191, 186

Störzer, H., \& Hollenbach, D. 1999, ApJ, 515, 669

Stolovy, S. R., Burton, M. G., Erickson, E. F., et al. 1998, ApJ, 492, L151

Sugai, H., Kawabata, H., Usuda, T., et al. 1995, ApJ, 442, 674

Timmermann, R. 1998, ApJ, 498, 246

Troland, T. H., \& Heiles, C. 1986, ApJ, 301, 339

Vannier, L., Lemaire, J. L., Field, D., et al. 2001, A\&A, 366, 651

Wilgenbus, D., Cabrit, S., Pineau des Forêts, G., \& Flower, D. R. 2000, A\&A, 356, 1010

Zapata, L. A., Rodríguez, L. F., Kurtz, S. E., \& O’Dell, C. R. 2004, AJ, 127, 2252 